\title{
Finite element methods for the non-linear mechanics of crystalline sheets and nanotubes
}

\author{
M. Arroyo ${ }^{\ddagger}$ and T. Belytschko*, \\ Department of Mechanical Engineering, Northwestern University, Evanston, IL 60208, U.S.A.
}

\begin{abstract}
SUMMARY
The formulation and finite element implementation of a finite deformation continuum theory for the mechanics of crystalline sheets is described. This theory generalizes standard crystal elasticity to curved monolayer lattices by means of the exponential Cauchy-Born rule. The constitutive model for a twodimensional continuum deforming in three dimensions (a surface) is written explicitly in terms of the underlying atomistic model. The resulting hyper-elastic potential depends on the stretch and the curvature of the surface, as well as on internal elastic variables describing the rearrangements of the crystal within the unit cell. Coarse grained calculations of carbon nanotubes (CNTs) are performed by discretizing this continuum mechanics theory by finite elements. A smooth discrete representation of the surface is required, and subdivision finite elements, proposed for thin-shell analysis, are used. A detailed set of numerical experiments, in which the continuum/finite element solutions are compared to the corresponding full atomistic calculations of CNTs, involving very large deformations and geometric instabilities, demonstrates the accuracy of the proposed approach. Simulations for large multi-million systems illustrate the computational savings which can be achieved. Copyright (c) 2003 John Wiley \& Sons, Ltd.
\end{abstract}

KEY WORDS: carbon nanotubes; continuum surface model; hyperelasticity; finite elements

\section{INTRODUCTION}

The growing interest in nanoscale science and engineering has provided a tremendous impetus for the development of numerical methods to simulate objects at those scales. Nanoscale mechanics are usually analysed by atomistic simulations [1-6]. The computational cost of such calculations limits them to relatively small systems for very short time intervals. For example, even for bundles or multi-walled nanotubes, the computational cost becomes prohibitive. Remarkably, for many problems of interest, even when the scales are of a few to

\footnotetext{
*Correspondence to: T. Belytschko, Department of Mechanical Engineering, Northwestern University, Evanston, IL 60208, U.S.A.

${ }^{\dagger}$ E-mail: tedbelytschko@northwestern.edu

$¥$ Current address: Graduate Aeronautical Laboratories, California Institute of Technology, Pasadena, CA 91125, U.S.A.
}

Contract/grant sponsor: NASA URETI BIMat; contract/grant number: NCC-1-02037

Contract/grant sponsor: Army Research Office; contract/grant number: DAAD19-02-1-0339

Received 14 February 2003

Revised 28 June 2003

Copyright (c) 2003 John Wiley \& Sons, Ltd.

Accepted 10 July 2003 
hundred nanometers, properly formulated continuum methods provide very accurate simulations as compared to atomistic calculations, as will become clear from the examples in this paper. These continuum based methods are much faster than molecular simulations for systems of engineering interest, which makes them attractive. Although the presence of defects does not rule out continuum models, the present work does not deal with them. The present paper addresses the space scale issue, and a coarse-grained computational approach for crystalline sheets based on continuum mechanics is developed and numerically verified.

Because of the wealth of experimental results on carbon nanotubes (CNTs), as well as their technological interest, we will focus on them in the applications. The ideas presented are applicable to other curved lattices of reduced dimensionality (see Reference [7] for applications to discrete chains). Carbon nanotubes can be viewed as single layer crystalline sheets rolled into cylinders, with diameters in the nanometer range. These large molecules, or small solids, possess exceptional mechanical, electronic, thermal, and chemical properties which have fostered an intense research in CNTs based nanostructured materials. Furthermore, deformation morphologies reminiscent of macroscopic objects such as buckled shells have been observed [8]. These severe deformations have been shown to be reversible $[9,10]$, resulting in remarkably resilient nanostructures. Several nanodevices have been proposed, which rely often on the strong dependence of other properties of CNTs, such as the electrical conductance, on the deformation $[11,12]$.

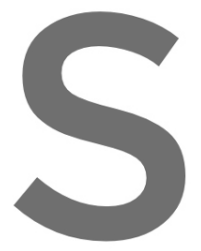

Continuum mechan tions because they do the atomic sites. Ma concepts to carbon a covalent-bonded syst cases, the continuum not need to track every
ny authors $[1,13-16]$ ha
anotubes, although. as no
stem of orily a few ator
models are phenomenolo atom, and the di
ve applied contin
ted by Yakobso
gical and restricted diameter i ystems than atomistic calcula-
iscretization is independent of
nuum or structural mechanics
et al. [1] 'jts relevance for
s far from obvious'. In most
ted to small deformations. Or occasions, the Euler-Bernoulli beam theory is adopted to model CNTs, and the adequacy of

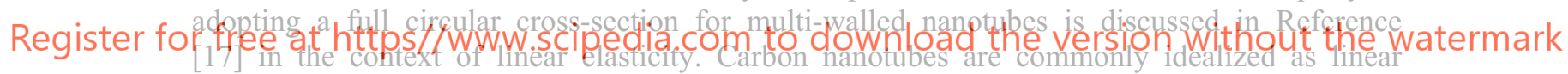
elastic thin shells $[1,18-22]$, with material parameters fit to available data. A first difficulty encountered by this approach is in the definition of a shell thickness for an intrinsicaliy twodimensional lattice such as the graphene monolayer (see Reference [23] for a discussion). Furthermore, nanotubes have been experimentally shown to undergo very large non-linear deformations elastically, i.e. reversibly, with intact bond topology [9-11], sometimes under very small loads [24]. Some authors have attempted to describe the mechanics of CNTs through non-linear elastic shell theory, by considering non-linear models which match the infinitesimal elastic constants obtained from atomistics or experiments $[25,26]$. However, the choice of a particular non-linear model cannot be justified, and this choice affects the response in the nonlinear regime. The aim of the present approach for the mechanics of crystalline monolayers is to circumvent phenomenological models of elasticity, i.e. avoid parameter fitting of any sort, and instead deductively construct a finite deformation continuum model from an atomistic model. An alternative asymptotic approach has been recently proposed by Friesecke and James [27]; Qian et al. [28] proposed a method based on constraining the atomic positions to remain on a surface represented by a meshfree approximation, in the spirit of the non-local quasicontinuum method.

The systematic approach used here, relying on kinematic rules relating the continuum and the discrete (atomistic) deformations, has a long and fruitful history. Molecular theories of 
elasticity bridge atomistic descriptions of crystalline solids with finite deformation continuum mechanics [29-35], and thus provide a natural framework to overcome the limitations of commonly used continuum models. The Cauchy-Born rule, also referred as method of the homogeneous deformations, is a fundamental kinematic assumption that links the deformation of lattice vectors to that of the continuous medium, and has been recently proven rigorously to hold under certain conditions [36]. By means of the Cauchy-Born rule, the continuum elastic potential can be obtained by equating the deformation energy of a representative cell of the lattice to that of an equivalent volume of the continuum. The resulting continuum constitutive model depends only on the interatomic interactions, without additional phenomenological input. Consequently, its ability to describe the physical system can only be as good the model used for the interatomic potential. Constitutive models constructed in this fashion inherit the crystal symmetries and anisotropy, and can treat finite deformations. Finite crystal elasticity has been used to obtain elastic moduli and study the stability of crystals $[29,37,38]$, to study phase transformations in solids $[35,39]$, and recently these ideas have been cast in a computational framework to solve general boundary value problems in combination with finite elements by the quasicontinuum method [40-43]. This method has emerged as an efficient and accurate simulation method at the nano-scale. The method implemented here is a generalization of the local quasicontinuum $[42,43]$ to curved crystalline monolayer sheets.

The basic kinematic law linking atomistic and continuum deformations, the Cauchy-Born

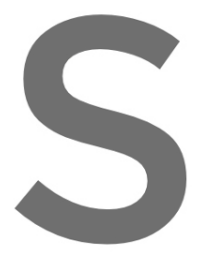
rule, cannot be appliect ticularly if curvature exponential Cauchylayer lattices. The resu spond with conventid thickness. (References 'as is'
effects
orn rule
ulting finit
al shell
$45,46\rceil$ to the case of
are to be account
which extends the
inite deformation
theories, and vid
presented contin curved crystalli
the standard Cau
continum me
cws crystalline
inuum models fo e sheets one atom thic
rence [44], we develo
hy -Born rule to curved
hanics model does not
onolayers as surfaces
CNTs based on crystat ticity, but viewed the tube wall as a shell with thickness.) This is why the term membrane

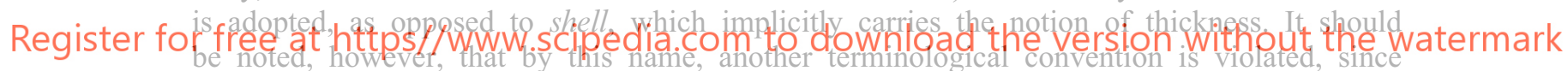
the elastic energy of a classical membrane depends only on its stretch; in the present theory, it depends also on the curvature, and thereby the membrane has bending stifiness as well. This model properly describes the rearrangements within the unit cell of multi-lattices by the introduction of a field of internal elastic variables.

In the present paper, the numerical implementation of the continuum model for curved crystalline sheets presented in Reference [44] is given. For completeness, the theory is also reviewed. Section 2 describes the kinematic setting, as well as the exponential Cauchy-Born rule relating the deformation of the surface to that of the lattice. In Section 3, for concreteness, the formulation is particularized to graphene and carbon nanotubes. The hyperelastic constitutive relation for graphene is constructed in terms of a bond-order potential (the Tersoff-Brenner potential). It does not require local atomistic calculations, i.e. it is written in closed-form. Details about its evaluation and the calculation of the corresponding stress measures are provided. A continuum version of the van der Waals interactions is also formulated, and the continuum statement for the statics of the membrane is provided. In Section 4, the finite element implementation of the theory is developed. Section 5 presents numerical simulations of a widely studied crystalline sheet, carbon nanotubes. They demonstrate that the continuum/finite element approach accurately reproduces the parent atomistic model in the full non-linear regime. Our simulations suggest that, in the absence of bond rearrangement or defects, the non-linear 
mechanics of curved crystalline sheets can be accurately modelled within the strict framework of continuum mechanics. Numerical examples also illustrate the dramatic computational savings which can be achieved for large multi-walled nanotubes containing millions of atoms, and replicate some unusual features observed in experiments.

\section{EXPONENTIAL CAUCHY-BORN RULE FOR 2D LATTICES}

This section presents a concise formulation of the kinematics of the surface replacing the curved single layer two-dimensional lattice. This presentation focuses on the practical computation of the variables of interest for a numerical implementation of the theory. Then, the local approximation of the exponential Cauchy-Born rule for surfaces is presented, which links the deformation of the atomic bonds to that of the continuum surface.

\section{Kinematics}

As argued in Reference [44], the continuum object replacing the crystalline monolayer is a surface without thickness. The nuclei are assumed to lie on the surface, and therefore, the bonds are chords of the surface. It is convenient to define the undeformed or reference system as the planar crystalline sheet. This choice is natural for carbon nanotubes, since this state

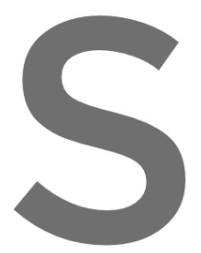
represents the groun [47], and Reference Reference [49] for a particularly with respect

\subsubsection{General setting, co-che}
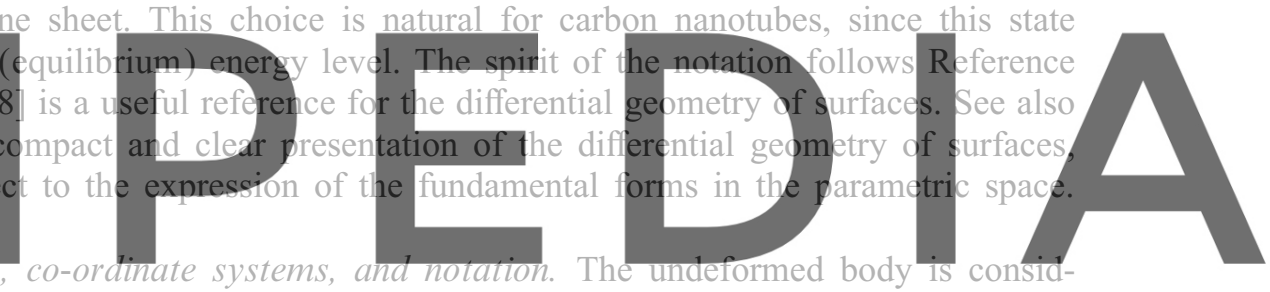

ered to be two-dimensional, i.e. $\Omega_{0}$ is an open set of $\mathbb{R}^{2}$, and models a slab of planar crys-

$X \in \Omega_{0} \longmapsto x=\Phi(X) \in \mathbb{R}^{3}$. The deformed body $\Omega=\Phi\left(\Omega_{0}\right)$ is a smooth surface. Let us describe the undeformed body by Euclidean co-ordinates $\left\{X^{1}, X^{2}\right\}$. The corresponding orthonormal basis of the tangent of the undeformed body $T \Omega_{0}$ is $\mathscr{B}_{0}=\left\{\mathbf{I}_{1}, \mathbf{I}_{2}\right\}$. Analogously, the Euclidean co-ordinates $\left\{x^{1}, x^{2}, x^{3}\right\}$ describe $\mathbb{R}^{3}$, and the associated standard basis is $\mathscr{B}=\left\{\mathbf{i}_{1}, \mathbf{i}_{2}, \mathbf{i}_{3}\right\}$.

It is convenient in the numerical formulation to define a parametric body $\bar{\Omega} \subset \mathbb{R}^{2}$. Let this parametric body be described by Euclidean co-ordinates $\left\{\xi^{1}, \xi^{2}\right\}$, and the corresponding orthonormal basis is $\overline{\mathscr{B}}=\left\{\boldsymbol{\Xi}_{1}, \boldsymbol{\Xi}_{2}\right\}$. The undeformed configuration is a differentiable and invertible map $\xi \in \bar{\Omega} \longmapsto X=\varphi_{0}(\xi) \in \Omega_{0} \subset \mathbb{R}^{2}$ such that $\varphi_{0}(\bar{\Omega})=\Omega_{0}$. Similarly, the deformed configuration maps smoothly and bijectively the parametric body into the deformed body $\xi \in \bar{\Omega} \longmapsto x=\varphi(\xi) \in \Omega \subset \mathbb{R}^{3}, \varphi(\bar{\Omega})=\Omega$. The deformation map is then

$$
\Phi=\varphi \circ \varphi_{0}^{-1}: X \in \Omega_{0} \longmapsto x=\Phi(X)=\varphi\left(\varphi_{0}^{-1}(X)\right) \in \Omega
$$

The vector from the origin to the point $\Phi(X)$ in $\mathbb{R}^{3}$ is denoted by $\boldsymbol{\Phi}(X)$, and coincides with the vector $\varphi(\xi)$ where $\xi=\varphi_{0}^{-1}(X)$. The components of these vectors, for instance $\boldsymbol{\varphi}$, in the standard basis $\mathscr{B}$ coincide with the components of the point mappings $\varphi$ in the co-ordinate system $\left\{x^{1}, x^{2}, x^{3}\right\}$, and are therefore denoted by the same symbol $\varphi^{a}, a=1,2,3$. Note that boldface is reserved for vectors and tensors, while points $(x, X, \xi, \ldots)$ and point mappings $\left(\Phi, \varphi_{0}, \varphi, \ldots\right)$ are denoted with lightface. This general setting is illustrated in Figure 1. 


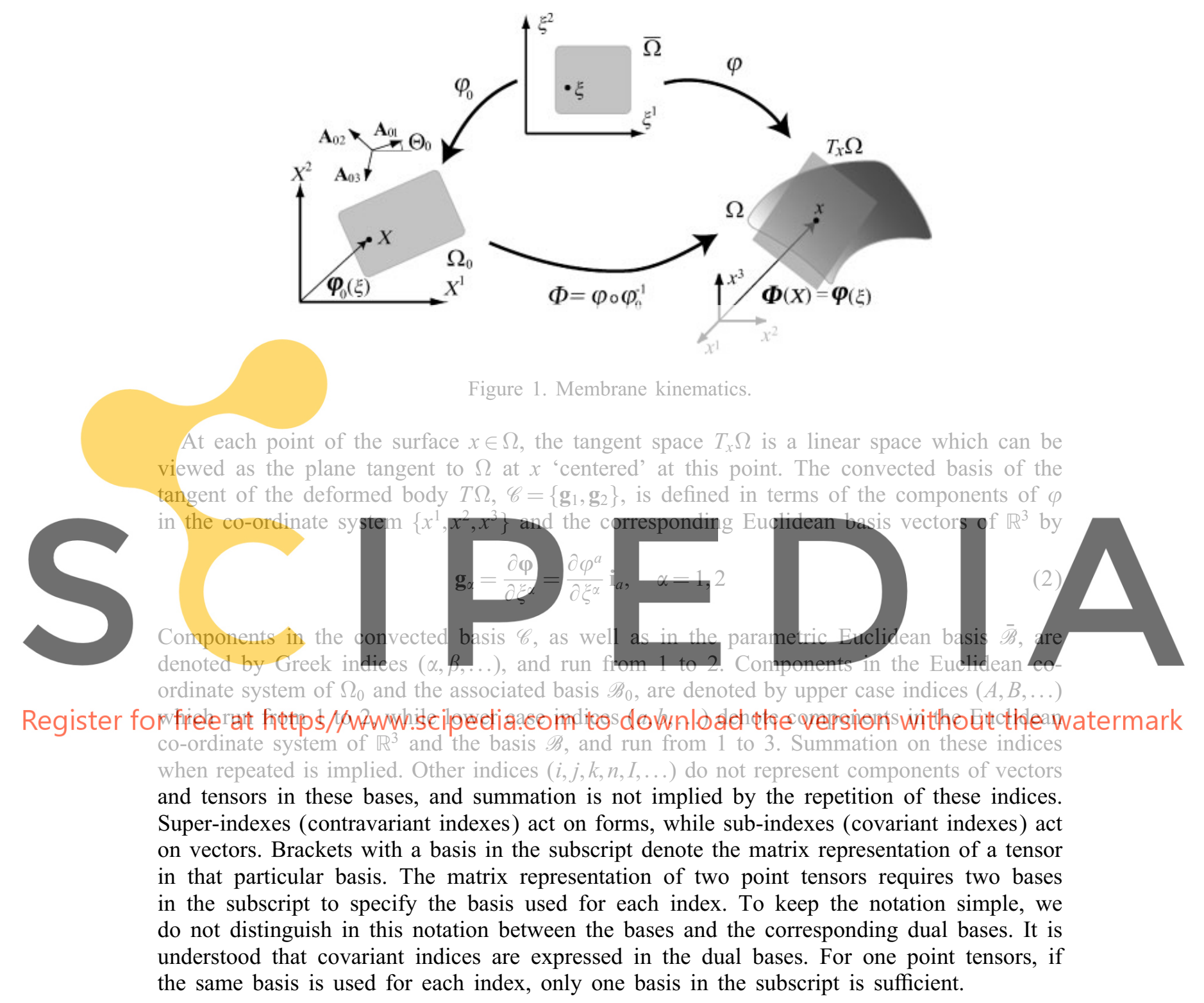

2.1.2. Tangents of the configurations and deformation gradient. In the following, indicial notation, invariant notation, as well as matrix representation of tensors are provided on many occasions for clarity. The matrix representation of the tangent map of the undeformed configuration in the Euclidean bases $\overline{\mathscr{B}}-\mathscr{B}_{0}$, is denoted according the our conventions as $\left[T \varphi_{0}\right]_{\mathscr{B}_{0}} \overline{\mathscr{B}}$. Its components can be computed as $\left(T \varphi_{0}\right)^{A}{ }_{\alpha}=\partial \varphi_{0}^{A} / \partial \xi^{\alpha}$. The area element in the undeformed body can be expressed as

$$
\mathrm{d} \Omega_{0}=\operatorname{det}\left[T \varphi_{0}\right]_{\mathscr{B}_{0} \overline{\mathscr{B}}} \mathrm{d} \xi^{1} \mathrm{~d} \xi^{2}
$$


On the other hand, noting that the image of the referential basis vector $\boldsymbol{\Xi}_{\alpha}$ through the tangent of the deformed configuration $T \varphi$ is the convected basis vector $\mathbf{g}_{\alpha}$, we have $[T \varphi]_{\mathscr{C} \bar{B}}=$ $[\mathbf{I d}]_{2 \times 2}$ (the information about the deformation is contained in the convected basis vectors).

Applying the chain rule, the deformation gradient can be written as $\mathbf{F}=T \Phi=T \varphi \circ T \varphi_{0}^{-1}$. For pull-back operations in the following sections, the components of the deformation gradient in the bases $\mathscr{B}_{0}-\mathscr{C}$ are needed. Thus, we have

$$
[\mathbf{F}]_{\mathscr{C}_{\mathscr{B}_{0}}}=[T \varphi]_{\mathscr{C} \overline{\mathscr{B}}}\left[T \varphi_{0}^{-1}\right]_{\overline{\mathscr{B}} \mathscr{B}_{0}}=[\mathbf{I d}]_{2 \times 2}\left[T \varphi_{0}\right]_{\mathscr{B}_{0} \mathscr{\mathscr { B }}}^{-1}=\left[T \varphi_{0}\right]_{\mathscr{B}_{0} \mathscr{\mathscr { B }}}^{-1}
$$

or in components

$$
F_{A}^{\alpha}=\delta^{\alpha}{ }_{\beta}\left(T \varphi_{0}^{-1}\right)_{A}^{\beta}=\left(T \varphi_{0}^{-1}\right)^{\alpha}{ }_{A}
$$

\subsubsection{The metric and the Green deformation tensors. The matrix representation of the metric} tensor of the surface $\Omega$ in the convected basis is:

$$
[\mathbf{g}]_{\mathscr{C}}=\left[\begin{array}{ll}
g_{11} & g_{12} \\
g_{21} & g_{22}
\end{array}\right]
$$

where the covariant components (the components in $\mathscr{C}$ ) are obtained from the convected basis vectors as
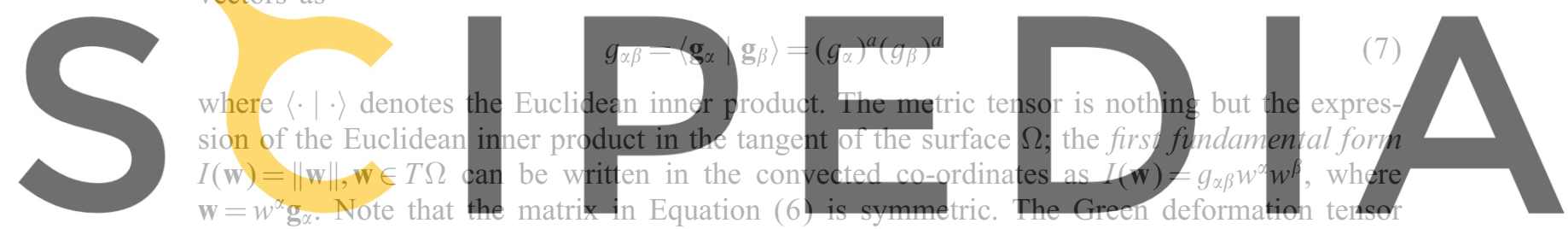

(with lowered indexes) is defined as the pull-back of the metric tensor $\mathbb{C}^{b}=\Phi^{*} \mathrm{~g}$. Therefore,

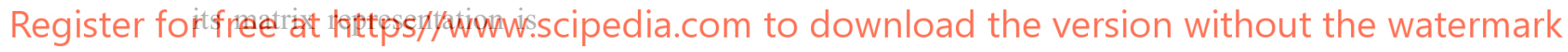

$$
\left[\mathrm{C}^{b}\right]_{\mathscr{B}_{0}}=[\mathbb{F}]_{\mathscr{C} \mathscr{B}_{0}}^{\mathrm{T}}[\mathrm{g}]_{\mathscr{C}}[\mathrm{F}]_{\mathscr{C} \mathscr{B}_{0},} \text { or } C_{A B}=g_{\alpha \beta \beta} F^{\alpha}{ }_{A} F^{\beta}{ }_{B}
$$

Thus, the matrix expression of the Green deformation tensor (with lowered indexes) in the basis $\mathscr{B}_{0}$ is the symmetric matrix:

$$
\left[\mathbf{C}^{b}\right]_{\mathscr{B}_{0}}=\left[T \varphi_{0}\right]_{\mathscr{B}_{0} \mathscr{\mathscr { B }}}^{-\mathrm{T}}[\mathbf{g}]_{\mathscr{C}}\left[T \varphi_{0}\right]_{\mathscr{B}_{0} \overline{\mathscr{B}}}^{-1}, \quad \text { or } \quad C_{A B}=g_{\alpha \beta}\left(T \varphi_{0}^{-1}\right)^{\alpha}{ }_{A}\left(T \varphi_{0}^{-1}\right)_{B}^{\beta}
$$

Since the Green deformation tensor (with lowered indexes) $\mathbf{C}^{b}$ is always expressed in the Euclidean basis $\mathscr{B}_{0}$, we do not need to distinguish between the tensors $\mathbf{C}^{b}$ and $\mathbf{C}$. To simplify the notation, the ${ }^{b}$ is dropped.

2.1.4. The principal curvatures. The unit normal to the deformed body $\Omega$ is

$$
\mathbf{n}=\frac{1}{\left\|\mathbf{g}_{1} \times \mathbf{g}_{2}\right\|} \mathbf{g}_{1} \times \mathbf{g}_{2}
$$

or in components

$$
n^{a}=\frac{\varepsilon_{b c}^{a}\left(g_{1}\right)^{b}\left(g_{2}\right)^{c}}{\sqrt{\varepsilon^{d}{ }_{e f} \varepsilon^{d}{ }_{g h}\left(g_{1}\right)^{e}\left(g_{2}\right)^{f}}\left(g_{1}\right)^{g}\left(g_{2}\right)^{h}}
$$


where $\|\cdot\|$ denotes the Euclidean norm, and $\varepsilon_{b c}^{a}$ is the permutation symbol. The covariant components of the curvature tensor, that is the matrix elements of $[\mathbf{k}]_{\mathscr{C}}$, can be obtained as

$$
k_{\alpha \beta}=\left\langle\mathbf{n} \mid \mathbf{g}_{\alpha, \beta}\right\rangle=n^{a}\left(g_{\alpha, \beta}\right)^{a}
$$

where $(\cdot)_{, \beta}$ denotes $\partial(\cdot) / \partial \xi^{\beta}$. The second fundamental form of the surface $\Omega$ can be expressed in convected co-ordinates as $I I(\mathbf{w})=k_{\alpha \beta} w^{\alpha} w^{\beta}$.

Similarly to the metric tensor, the pull-back of the curvature tensor $\mathscr{K}=\Phi^{*} \mathbf{k}$ can be expressed in the Euclidean basis $\mathscr{B}_{0}$ as the symmetric matrix:

$$
[\mathscr{K}]_{\mathscr{B}_{0}}=\left[T \varphi_{0}\right]_{\mathscr{B}_{0} \overline{\mathscr{B}}}^{-\mathrm{T}}[\mathbf{k}]_{\mathscr{C}}\left[T \varphi_{0}\right]_{\mathscr{B}_{0} \overline{\mathscr{B}}}^{-1}, \quad \text { or } \quad \mathscr{K}_{A B}=k_{\alpha \beta}\left(T \varphi_{0}^{-1}\right)^{\alpha}{ }_{A}\left(T \varphi_{0}^{-1}\right)^{\beta}{ }_{B}
$$

The principal curvatures $k_{1}$ and $k_{2}$, and the principal directions $\mathbf{v}_{1}$ and $\mathbf{v}_{2}$ of the surface $\Omega$ are the eigenvalues and eigenvectors of the Weingarten map, i.e. they are characterized by being the maximum and the minimum of the quotient $I I(\mathbf{w}) / I(\mathbf{w}), \mathbf{w} \in T \Omega$ and $\mathbf{w} \neq \mathbf{0}$. Note that the principal directions are tangent to the surface. Using convected co-ordinates, the principal curvatures and directions are found as solutions of the generalized eigenvalue problem

$$
[\mathbf{k}]_{\mathscr{C}}[\mathbf{v}]_{\mathscr{C}}=k[\mathbf{g}]_{\mathscr{C}}[\mathbf{v}]_{\mathscr{C}}
$$

(14)

Alternatively, it is possible to find the principal curvatures, and the pull-back of the principal
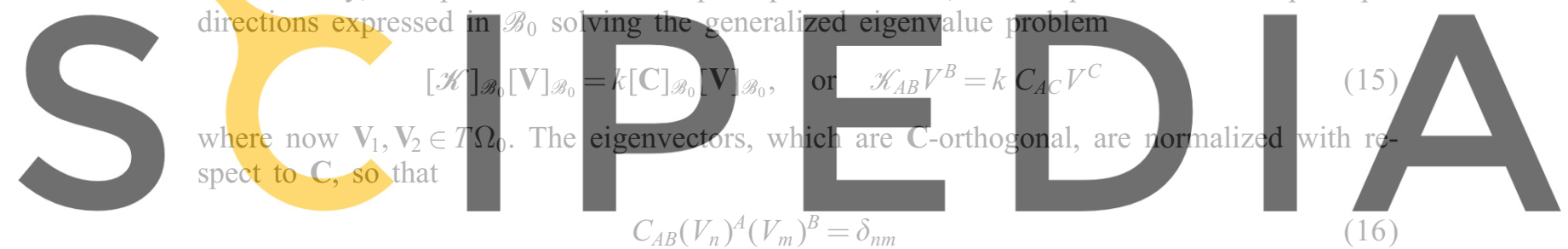

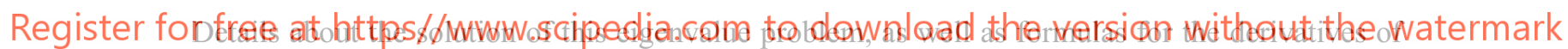

the principal curvatures and directions with respect to $\mathbf{C}$ and $\mathscr{K}$ are provided in Appendix A.1.

\subsection{Local approximation of the exponential Cauchy-Born rule}

As detailed in Reference [44], the standard Cauchy-Born rule postulates that the lattice vectors deform according to the linear transformation $\mathbf{a}=\mathbf{F}(X) \mathbf{A}$, where $\mathbf{A}$ denotes an undeformed lattice vector emanating from $X$ and a denotes this vector after deformation. This kinematic assumption, which for space-filling crystals has proven to be useful and rigorously valid in some cases, fails to capture the mechanics of curved crystalline monolayers. Inspired by the geometric structure of the Cauchy-Born rule in the context of the finite deformation kinematics of surfaces, an extension of the Cauchy-Born rule to account for the curvature of the film was proposed in Reference [44]. The exponential Cauchy-Born rule can be summarized with the formula $\mathbf{a}=\exp \circ \mathbf{F}(X) \mathbf{A}$, where exp denotes the exponential map of the surface at $\Phi(X)$ [48]. The basic idea is that, in the setting of $2 \mathrm{D}$ lattices deforming in $3 \mathrm{D}$, the standard Cauchy-Born rule produces a deformed lattice vector which is tangent to the surface, not a chord (recall that we view atomic bonds as chords to the surface). The exponential map brings this tangent vector back to the surface, thereby defining a chord (see Figure 2 for an illustration). See Reference [50] for the simplest application of this idea to an atomic chain deforming in $2 \mathrm{D}$. 


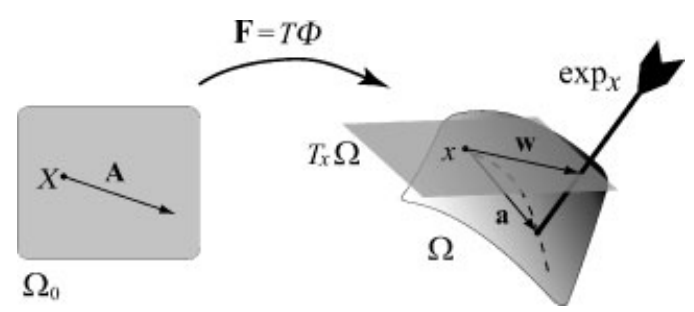

Figure 2. The exponential map transforms the vector $\mathbf{w}=\mathbf{F A}$ tangent to the surface into a chord of the surface a.

In general the evaluation of the exponential map, and therefore the application of the exponential Cauchy-Born rule, requires the knowledge of the geodesic curves. In a given co-ordinate system, these are obtained by integration of a system of two non-linear ODEs. The coefficients of these equations are the Christoffel symbols. In general, a closed-form solution of these equations is not available, and the exponential Cauchy-Born rule must be approximated in order to obtain practical local models. An approximation based on decoupling the principal directions was proposed in Reference [44]. The final formula is presented next. This approach is simple and leads to accurate models, as demonstrated by the numerical experiments described later.
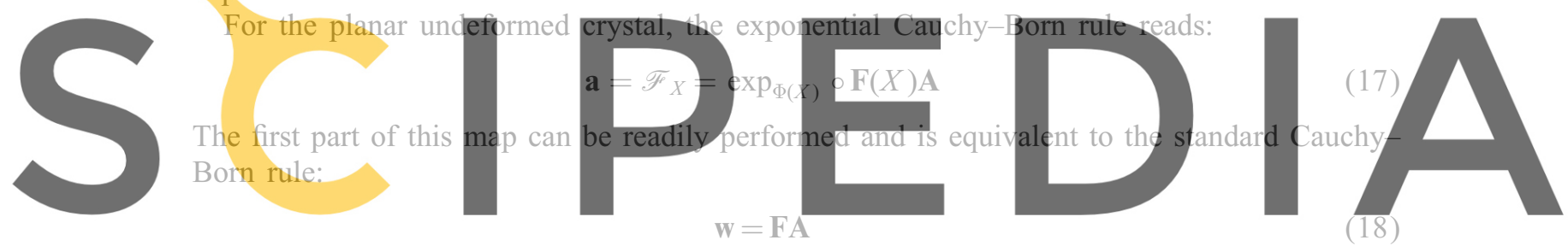

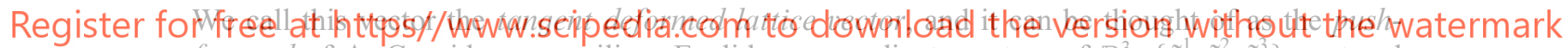
forward of $\mathbf{A}$. Consider an auxiliary Euclidean co-ordinate system of $\mathbb{R}^{3},\left\{\tilde{x}^{1}, \tilde{x}^{2}, \tilde{x}^{3}\right\}$ centered at $x=\Phi(X)$ and whose axes are parallel to $\mathbf{v}_{1}, \mathbf{v}_{2}$, and $\mathbf{v}_{1} \times \mathbf{v}_{2}$. The associated orthonormal basis is $\tilde{\mathscr{B}}=\left\{\mathbf{v}_{1}, \mathbf{v}_{2}, \mathbf{v}_{1} \times \mathbf{v}_{2}\right\}$. Consider also the restriction of this co-ordinate system to $T_{x} \Omega,\left\{\tilde{x}^{1}, \tilde{x}^{2}\right\}$ with the basis $\tilde{\mathscr{B}}_{T_{x} \Omega}=\left\{\mathbf{v}_{1}, \mathbf{v}_{2}\right\}$. The components of $\mathbf{w}$ in the basis $\tilde{\mathscr{B}}_{T_{x} \Omega}$ can be computed as

$$
\left\{\begin{array}{l}
w^{1} \\
w^{2}
\end{array}\right\}=\left\{\begin{array}{l}
\left\langle\mathbf{w} \mid \mathbf{v}_{1}\right\rangle \\
\left\langle\mathbf{w} \mid \mathbf{v}_{2}\right\rangle
\end{array}\right\}=\left\{\begin{array}{l}
\mathbf{V}_{1} \cdot \mathbf{C} \cdot \mathbf{A} \\
\mathbf{V}_{2} \cdot \mathbf{C} \cdot \mathbf{A}
\end{array}\right\}=\left\{\begin{array}{l}
C_{A B} A^{A}\left(V_{1}\right)^{B} \\
C_{A B} A^{A}\left(V_{2}\right)^{B}
\end{array}\right\}
$$

By defining $2(x)=\sin x / x$, the expression for the deformed lattice vector in the orthonormal basis $\tilde{\mathscr{B}}$ provided by the local approximation to the exponential Cauchy-Born rule is

$$
[\mathbf{a}]_{\tilde{B}}=\left\{\begin{array}{c}
a^{1} \\
a^{2} \\
a^{3}
\end{array}\right\}=\left\{\begin{array}{c}
w^{1} \mathscr{2}\left(k_{1} w^{1}\right) \\
w^{2} \mathscr{2}\left(k_{2} w^{2}\right) \\
\frac{k_{1}\left(w^{1}\right)^{2}}{2} \mathscr{Q}^{2}\left(k_{1} w^{1} / 2\right)+\frac{k_{2}\left(w^{2}\right)^{2}}{2} \mathscr{Q}^{2}\left(k_{2} w^{2} / 2\right)
\end{array}\right\}
$$

Bearing Equation (18) in mind, and the fact that $k_{1,2}$ and $\mathbf{V}_{1,2}$ are obtained from the eigenvalue problem (15), it is clear that $[\mathbf{a}]_{\tilde{\mathscr{B}}}$ depends only on the undeformed lattice vector $\mathbf{A}$, the Green deformation tensor $\mathbf{C}$ and the pull-back of the curvature tensor $\mathscr{K}$. 
The length of a deformed bond, and the angle between two deformed bonds a and $\mathbf{b}$ obtained following Equation (20) can be computed simply as

$$
a=\|\mathbf{a}\|=\sqrt{a^{c} a^{c}} \quad \text { and } \quad \theta=\arccos \frac{\langle\mathbf{a} \mid \mathbf{b}\rangle}{a b}=\arccos \frac{a^{c} b^{c}}{a b}
$$

Thus, the bond lengths and angles have been expressed in terms of the continuum strain measures $\mathbf{C}$ and $\mathscr{K}$. Basically, we have obtained the derived strain measures $a=f(\mathbf{C}, \mathscr{K} ; \mathbf{A})$ and $\theta=g(\mathbf{C}, \mathscr{K} ; \mathbf{A}, \mathbf{B})$. These continuous strain measures are adequate to formulate continuum models from the atomistic description of the system, given both the lattice structure and the interatomic potential. Note that, although here the potential depends on the bond lengths and angles, it is straightforward to apply the methodology presented here to atomistic descriptions which include dihedral angles.
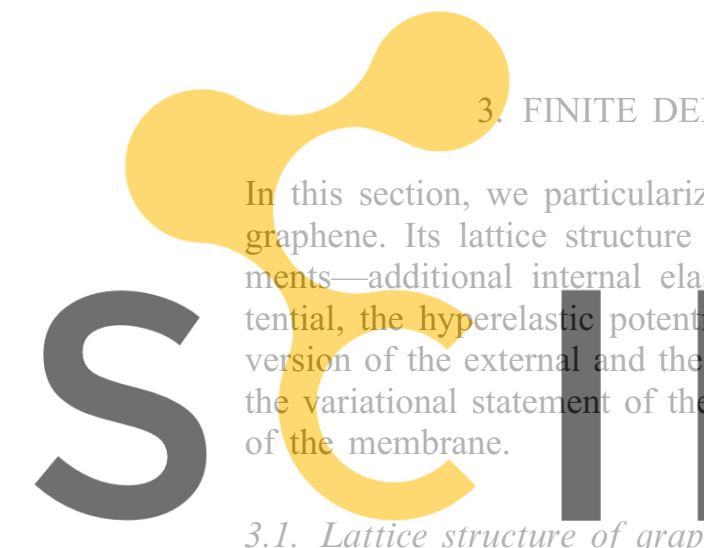

3. FINITE DEFORMATION MEMBRANE FOR NANOTUBES

In this section, we particularize the formulation to a representative crystalline sheet, namely graphene. Its lattice structure is described, and the need to account for the inner displacements - additional internal elastic variables - is highlighted. Then, given an interatomic potential, the hyperelastic potential for the continuum membrane is formulated. The continuum
version of the external and the non-bonded potential is then presented. With these ingredients,
the variational statement of the continum boundary value problem is provided for the statics
of the membrane.
3.1. Lattice structure of graphene

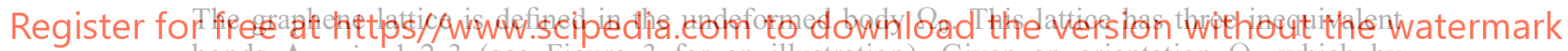
bonds $\mathbf{A}_{0 i}, i=1,2,3$ (see Figure 3 for an illustration). Given an orientation $\Theta_{0}$ which by symmetry considerations takes values in $(-\pi / 6, \pi / 6]$, these undeformed bond vectors can be defined as

$$
\begin{aligned}
& {\left[\mathbf{A}_{01}\right]_{\mathscr{B}_{0}}=A_{0}\left\{\begin{array}{l}
\cos \Theta_{0} \\
\sin \Theta_{0}
\end{array}\right\}} \\
& {\left[\mathbf{A}_{02}\right]_{\mathscr{B}_{0}}=A_{0}\left\{\begin{array}{l}
\cos \left(\Theta_{0}+2 \pi / 3\right) \\
\sin \left(\Theta_{0}+2 \pi / 3\right)
\end{array}\right\}} \\
& {\left[\mathbf{A}_{03}\right]_{\mathscr{B}_{0}}=A_{0}\left\{\begin{array}{l}
\cos \left(\Theta_{0}-2 \pi / 3\right) \\
\sin \left(\Theta_{0}-2 \pi / 3\right)
\end{array}\right\}}
\end{aligned}
$$

where $A_{0}$ is the equilibrium bond length (see Figure 1). When modelling nanotubes, the initial deformed configuration is a cylindrical surface, and thus the initial deformation map maps the undeformed planar graphene sheet into this cylinder. The chirality in the tube can be specified by selecting the appropriate orientation $\Theta_{0}$ with respect to the rolling orientation. For example, suppose a $\left(n_{1}, n_{2}\right)$ nanotube is modelled. Its ideal radius is given 


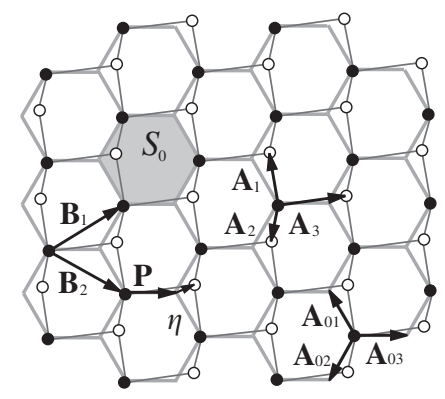

Figure 3. Graphene honeycomb multi-lattice: the two simple Bravais lattices, depicted in different colors, are relatively displaced by the inner displacement $\eta$, which conseguently also affects the bond vectors which are transformed from $\mathbf{A}_{0 i}$ into $\mathbf{A}_{i}$. The unit cell of area $S_{0}$ is also represented. It contains two nuclei and three inequivalent bonds.

by $R_{0}=A_{0} \sqrt{3\left(n_{1}^{2}+n_{1} n_{2}+n_{2}^{2}\right)} / 2 \pi$, and the chiral angle by $\arctan \left[\sqrt{3} n_{2} /\left(2 n_{1}+n_{2}\right)\right]$ [51]. In this situation, the undeformed body for such a nanotube of length $L$ can be defined in $\left\{X^{1}, X^{2}\right\}$ as $\Omega_{0}=(0, L) \times\left(0,2 \pi R_{0}\right)$. The initial deformed configuration of the nanotube bringing this undeformed body into the initial cylinder of length $L$ can be defined in $\left\{x^{1}, x^{2}, x^{3}\right\}$ as $\Phi^{1}=X^{1}, \Phi^{2}=R_{0} \cos \left(X^{2} / R_{0}\right)$, and $\Phi^{3}=R_{0} \sin \left(X^{2} / R_{0}\right)$. In this situation, the chiral angle coin-

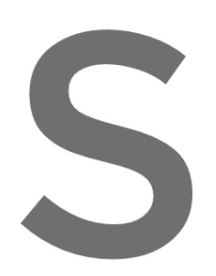
cides with $\Theta_{0}$. Thus, a zig-zag nanotube is characterized in th
arm-chair nanotube by $\Theta_{0}=\pi / 6$.
When dealing with graphene, special attention must be paid to
lattice is a Bravais nulti-lattice. These lattices have more than
viewed as a collection of inter-penetrating simple rattices (see $F$ site (say a black one) and the lattice basis vectors $\mathbb{B}_{1}$ and $\mathbb{B}_{2}$, are not enough to define the entire lattice, in particular the white sites. Either a white site or the shift vector $\mathbb{P}$ is also

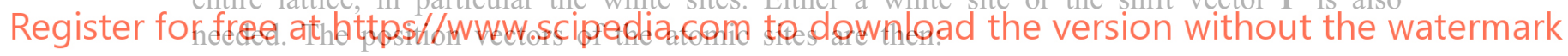

$$
\mathbf{X}_{n}=n^{i} \mathbf{B}_{i}+m \mathbb{P}
$$

where summation on the index $i$ is implied, $n^{1}$ and $n^{2}$ are integers, and $m$ takes the values 0 (black) or 1 (white).

The standard crystal elasticity treatment of multi-lattices is to assume that the homogeneous deformation affects each of the simple lattices. Additional kinematic variables describing the relative shifts of the simple lattices must be introduced to properly describe the configurations of uniformly strained multi-lattices. These relative shifts are called inner displacements $[29-32,42]$. The optical modes are the analog of the inner displacements in lattice dynamical theories [34]. The relative displacement of the basis nuclei cannot be represented by a homogeneous deformation, and is instead an internal mode of deformation. It is clear from Figure 3 that a perturbation in the shift vector by $\boldsymbol{\eta}$ leaves the basis vectors unchanged, but changes the configuration of the lattice by perturbing the triplet of bond vectors $\mathbf{A}_{0 i}$ by the same amount.

In the continuum setting, additional kinematic variables must be introduced to account for these rearrangements within the unit cell, which for graphene simply affect its elasticity; for other materials they may describe phase transformations [42,52]. Let $\boldsymbol{\eta}$ denote the inner displacements, which following Reference [42], are defined in the undeformed body, previous 
to the 'macroscopic' deformation $\Phi ; \boldsymbol{\eta}$ is a vector field in $T \Omega_{0}$. This guarantees rotational invariance of this kinematic variable. Owing to the inner displacements, the undeformed lattice vectors in $T \Omega_{0}$ become

$$
\mathbf{A}_{i}=\mathbf{A}_{0 i}+\boldsymbol{\eta}, \quad i=1,2,3
$$

A given continuum deformation transforms the triplet of undeformed bond vectors according to the exponential Cauchy-Born rule: $\mathbf{a}_{i}=\mathscr{F}_{X}\left(\mathbf{A}_{i}\right)=\mathscr{F}_{X}\left(\mathbf{A}_{0 i}+\boldsymbol{\eta}\right)$. Through Equations (20) and (21), it is possible to express the derived strain measures in terms of the local deformation of the surface and the inner displacements. Thus, the lengths of these deformed bond vectors can be written as

$$
a_{i}=f\left(\mathbf{C}, \mathscr{K} ; \mathbf{A}_{i}\right)=\bar{f}\left(\mathbf{C}, \mathscr{K}, \boldsymbol{\eta} ; \mathbf{A}_{0 i}\right), \quad i=1,2,3
$$

and the three angles these inequivalent bonds form after deformation as

$$
\theta_{i}=g\left(\mathbf{C}, \mathscr{K} ; \mathbf{A}_{j}, \mathbf{A}_{k}\right)=\bar{g}\left(\mathbf{C}, \mathscr{K}, \boldsymbol{\eta} ; \mathbf{A}_{0 j}, \mathbf{A}_{0 k}\right), \quad i=1,2,3
$$

where $\{i, j, k\}$ is an even permutation of $\{1,2,3\}$. The dependence on the inner displacements field through Equation (24) has been emphasized.

\subsection{Interatomic potential and constitutive model}

Once the lattice structure of the crystalline sheet has been described, and characterized in the continuum setting, a model for the potential energy of the atomistic system is needed. The Tersoff-Brenner potential for hydrocarbons [53] is considered in the simulations in the present paper, which follows the bond-order formalism [54]. This analytical potential has been widely used for carbon nanotubes [1,55], and expresses the energy in terms of bond lengths and angles, as a sum over the bonds:

$$
E=\sum_{i} \sum_{j>i}\left[V_{R}\left(r_{i j}\right)-\bar{B}_{i j} V_{A}\left(r_{i j}\right)\right]
$$

where $\bar{B}_{i j}$ depends on the lengths of the bonds and angles adjacent to the $i j$ th bond. Note that the present approach is not limited to analytical potentials (Reference [42] presented a quasicontinuum method based on the tight-binding method, while in Reference [52] an $a b$ initio Hamiltonian was considered).

By considering a representative cell, which for the graphene honeycomb lattice is hexagonal, contains two nuclei, one of each of the inequivalent bonds, and has a surface area of $S_{0}=(3 \sqrt{3} / 2) A_{0}^{2}$ (see Figure 3 ), the strain energy density (energy per unit area) of the continuum membrane can be written by dividing the energy of this cell by its area. For instance, for the Tersoff-Brenner potential it is

$$
W=W(\mathbf{C}, \mathscr{K}, \boldsymbol{\eta})=\frac{1}{S_{0}} \sum_{i=1}^{3}\left[V_{R}\left(a_{i}\right)-\bar{B}\left(a_{j}, a_{k}, \theta_{j}, \theta_{k}\right) V_{A}\left(a_{i}\right)\right]
$$

where $\{i, j, k\}$ is an even permutation of $\{1,2,3\}$. The dependence of this hyperelastic potential on the stretch $\mathbf{C}$ and curvature $\mathscr{K}$ of the surface, and on the inner displacement field $\boldsymbol{\eta}$ can be traced in Equations (25) and (26). The dependence of the energy on the undeformed lattice 
vectors has been omitted. Note that these strain measures and the definition of the inner displacements in the undeformed body guarantee frame indifference-rotational invarianceof the hyperelastic potential.

The inner displacements can be eliminated at the constitutive level. Given a deformation of the surface, the strain energy density can be minimized with respect to $\eta$ :

$$
\hat{\boldsymbol{\eta}}(\mathbf{C}, \mathscr{K})=\left.\arg \left(\min _{\boldsymbol{\eta}} W(\mathbf{C}, \mathscr{K}, \boldsymbol{\eta})\right) \Longrightarrow \frac{\partial W}{\partial \boldsymbol{\eta}}\right|_{\boldsymbol{\eta}=\hat{\boldsymbol{\eta}}}=\mathbf{0}
$$

After this inner relaxation, the strain energy density can be written as a function of only $\mathbf{C}$ and $\mathscr{K}$ :

$$
\hat{W}(\mathbf{C}, \mathscr{K})=W(\mathbf{C}, \mathscr{K}, \hat{\boldsymbol{\eta}}(\mathbf{C}, \mathscr{K}))
$$

Note that, while a closed-form expression for the hyperelastic potential $W$ is available (see Equation (28)), the evaluation of $\hat{W}(\mathbf{C}, \mathscr{K})$ involves a bivariate minimization problem, which is solved numerically by Newton's method (see Appendix A.2).

Two stress tensors (membrane and bending) can be defined by taking derivatives of the elastic potential with respect to the strain measures. As noted by Reference [42], in doing so one can benefit from the fact that the inner displacements are in internal equilibrium. Indeed, for the derivative with respect the stretch and using Equation (29), we have

$$
\frac{\partial \hat{W}}{\partial \mathbf{C}}=\left.\left(\frac{\partial W}{\partial \mathbf{C}}+\frac{\partial W}{\partial \eta^{A}} \frac{\partial \hat{\eta}^{A}}{\partial \mathbf{C}}\right)\right|_{\boldsymbol{\eta}=\hat{\boldsymbol{\eta}}}=\left.\frac{\partial W}{\partial \mathbf{C}}\right|_{\boldsymbol{\eta}=\hat{\boldsymbol{\eta}}}
$$

and therefore this derivative can be computed in closed-form from the function $W$.

The membrane or second Piola-Kirchhoff stress tensor is defined as

$$
\mathbf{S}=2 \frac{\partial \hat{W}}{\partial \mathbf{C}}=\left.2 \frac{\partial W}{\partial \mathbf{C}}\right|_{\boldsymbol{\eta}=\hat{\boldsymbol{\eta}}}
$$

and similarly, a Lagrangian bending (symmetric) tensor is defined as

$$
\mathbf{m}=\frac{\partial \hat{W}}{\partial \mathscr{K}}=\left.\frac{\partial W}{\partial \mathscr{K}}\right|_{\boldsymbol{\eta}=\hat{\boldsymbol{\eta}}}
$$

Box 1 , in combination with the appendices, describes the calculation of the strain energy density and the stresses. Note that the membrane stress tensor $\mathbf{S}$ has units of force divided by length (surface tension), while $\mathbf{m}$ has units of force. These stresses are not stress resultants, and the unusual units follow from the fact that the continuum object is a surface without thickness. 
Box 1. Constitutive model: calculation of the strain energy density and the stresses.

Given $C_{A B}$ and $\mathscr{K}_{B C}$,

1. Principal curvatures: Solve the eigenvalue problem of Equation (15), and obtain the principal curvatures, the pull-back of the principal directions, and their derivatives with respect to the strain measures (see Appendix A.1):

$$
k_{n}, \frac{\partial k_{n}}{\partial C_{A B}}, \quad \frac{\partial k_{n}}{\partial \mathscr{K}_{A B}},\left(V_{n}\right)^{A}, \frac{\partial\left(V_{n}\right)^{A}}{\partial C_{B C}}, \frac{\partial\left(V_{n}\right)^{A}}{\partial \mathscr{K}_{B C}}, n=1,2
$$

2. Inner relaxation: Minimize $W(\mathbf{C}, \mathscr{K}, \boldsymbol{\eta})$ with respect to $\boldsymbol{\eta}$, and find (see Appendix A.2):

- $\hat{\boldsymbol{\eta}}$ : relaxed inner displacements

- $\hat{W}$ : relaxed strain energy density

- $\left(A_{i}\right)^{A}=\left(A_{0 i}\right)^{A}+\hat{\eta}^{A}, i=1,2,3$ : updated undeformed lattice

3. Exponential Cauchy-Born rule: Compute bond lengths and angles (see Equations (20), (21), (25) and (26)), and their derivatives with respect to the strain measures (see Appendix A.3):

$$
a_{i}, \frac{\partial a_{i}}{\partial C_{A B}}, \frac{\partial a_{i}}{\partial \mathscr{K}_{A B}}, \theta_{i}, \frac{\partial \theta_{i}}{\partial C_{A B}}, \frac{\partial \theta_{i}}{\partial \mathscr{K}_{A B}}
$$

4. Stress tensors: Apply the chain rule to Equation (28), and recall Equations (32) and (33):

$$
\begin{aligned}
S^{A B} & =2 \sum_{i=1}^{3}\left(\frac{\partial W}{\partial a_{i}} \frac{\partial a_{i}}{\partial C_{A B}}+\frac{\partial W}{\partial \theta_{i}} \frac{\partial \theta_{i}}{\partial C_{A B}}\right) \\
m^{A B} & =\sum_{i=1}^{3}\left(\frac{\partial W}{\partial a_{i}} \frac{\partial a_{i}}{\partial \mathscr{K}_{A B}}+\frac{\partial W}{\partial \theta_{i}} \frac{\partial \theta_{i}}{\partial \mathscr{K}_{A B}}\right)
\end{aligned}
$$

\subsection{Non-bonded interaction and external forces}

The non-bonded or van der Waals interactions are generally treated by interatomic potentials that only act between non-bonded pairs of atoms. These diffuse interactions are critical to the mechanics of nanotubes interacting with substrates or packed in bundles, of multi-walled nanotubes, and of nanotubes in their collapsed configurations. The non-bonded energy of the atomistic system can be written as

$$
E_{\mathrm{nb}}=\sum_{i} \sum_{j>i, j \notin B_{i}} V_{\mathrm{nb}}\left(r_{i j}\right)=\frac{1}{2} \sum_{i} \sum_{j \notin B_{i}} V_{\mathrm{nb}}\left(r_{i j}\right)
$$

where $V_{\mathrm{nb}}$ is the non-bonded potential, $r_{i j}$ is the distance between atoms $i$ and $j$, and $B_{i}$ is the set of atoms bonded to atom $i$. A simple argument involving two representative cells of area $S_{0}$ each containing $n$ nuclei ( $n=2$ for graphene, see Figure 3 ) allows us to write the 
continuum van der Waals energy double density as

$$
\mathscr{V}_{\mathrm{nb}}(d)=\left(\frac{n}{S_{0}}\right)^{2} V_{\mathrm{nb}}(d)
$$

where $d$ is the distance between two points in the deformed body. The continuum counterpart of the total non-bonded energy takes then the form

$$
\Pi_{\mathrm{nb}}[\Phi]=\frac{1}{2} \int_{\Omega_{0}} \int_{\Omega_{0}-B_{X}} \mathscr{V}_{\mathrm{nb}}(\|\boldsymbol{\Phi}(X)-\boldsymbol{\Phi}(Y)\|) \mathrm{d} \Omega_{0 Y} \mathrm{~d} \Omega_{0 X}
$$

where $B_{X}$ is a ball centred at $X$ with a radius that is a function of the potential cut-off radius to account for the fact that this potential does not affect bonded atoms. The classical Lennard-Jones potential has been adopted here for the non-bonded interactions [56].

When external forces are applied on the nuclei (e.g. electrostatic forces), the continuum counterpart is a body force, and the corresponding potential energy is

$$
\Pi_{\mathrm{ext}}[\Phi]=\int_{\Omega_{0}} \mathbf{B} \cdot \boldsymbol{\Phi} \mathrm{d} \Omega_{0}
$$

where $\mathbf{B}$ is the body force per unit undeformed area.

\subsection{Boundary value problem}

From the developments of the previous section, we can express the internal energy of an elastic membrane whose undeformed configuration is a planar body $\Omega_{0}$, and which is subject to the deformation map $\Psi$, as

$$
\Pi_{\text {int }}[\Psi]=\int_{\Omega_{0}} \hat{W}(\mathbf{C}(\Psi), \mathscr{K}(\Psi)) \mathrm{d} \Omega_{0}
$$

The total potential energy of the system is then

$$
\Pi[\Psi]=\Pi_{\mathrm{int}}[\Psi]-\Pi_{\mathrm{ext}}[\Psi]+\Pi_{\mathrm{nb}}[\Psi]
$$

The stable equilibrium deformation maps of the system are given by

$$
\Phi=\arg \left(\inf _{\Psi \in \mathscr{C}} \Pi[\Psi]\right)
$$

i.e. the equilibrium deformation is a minimizer of $\Pi$. $\mathscr{C}$ is the appropriate space of deformation maps or trial functions accounting for essential boundary conditions, see e.g. Reference [57]. According to the principle of stationary energy, the equilibrium configurations of the system $\Phi$ are stationary points of the potential energy functional, and verify the principle of virtual work:

$$
0=\delta \Pi[\Phi ; \delta \Phi]=\int_{\Omega_{0}}\left(\frac{1}{2} \mathbf{S}: \delta \mathbf{C}+\mathbf{m}: \delta \mathscr{K}\right) \mathrm{d} \Omega_{0}-\delta \Pi_{\text {ext }}[\Phi ; \delta \Phi]+\delta \Pi_{\text {nb }}[\Phi ; \delta \Phi]
$$

for all $\delta \Phi \in \mathscr{V}$, the corresponding space of admissible variations. The expressions of $\delta \mathbf{C}$ and $\delta \mathscr{K}$ in terms of $\delta \Phi$ are described in Appendix B. The variations of the non-bonded and the 
external energy functionals are

$$
\begin{aligned}
\delta \Pi_{\mathrm{nb}}[\Phi ; \delta \Phi]= & \frac{1}{2} \int_{\Omega_{0}} \int_{\Omega_{0}-B_{X}} \frac{1}{\|\boldsymbol{\Phi}(X)-\boldsymbol{\Phi}(Y)\|} \mathscr{V}_{n b}^{\prime}(\|\mathbf{\Phi}(X)-\boldsymbol{\Phi}(Y)\|) \\
& \times\langle\boldsymbol{\Phi}(X)-\mathbf{\Phi}(Y) \mid \delta \boldsymbol{\Phi}(X)-\delta \boldsymbol{\Phi}(Y)\rangle \mathrm{d} \Omega_{0 Y} \mathrm{~d} \Omega_{0 X}
\end{aligned}
$$

and

$$
\delta \Pi_{\mathrm{ext}}[\Phi ; \delta \Phi]=\int_{\Omega_{0}} \mathbf{B} \cdot \delta \boldsymbol{\Phi} \mathrm{d} \Omega_{0}
$$

\section{NUMERICAL IMPLEMENTATION}

This section describes the numerical approximation of the above boundary value problem for the hyperelastic membrane. The configurations are approximated by a finite element scheme. Since the energy is a function of the curvature, the finite element space must have bounded second order derivatives. The calculation of the energy and the nodal forces, required by the minimization algorithm, is detailed.

\subsection{Finite element approximation}

Let us define the following notation: the superscript $(\cdot)^{h}$ denotes discretized finite element fields, as well as nodal values in global numbering, while the superscript $(\cdot)^{e}$ denotes the restriction to element $e$ of a finite element field or nodal values in local element numbering. The correspondence between these global and the local numbering schemes is established through the standard scatter and gather operations [57].

4.1.1. Configurations. In the finite element setting, the undeformed domain is triangulated in nel elements so that $\Omega_{0}=\bigcup_{e=1}^{\text {nel }} \mathscr{T}_{0}^{e}$ and $\mathscr{T}_{0}^{e} \cap \mathscr{T}_{0}^{f}=\varnothing$ if $e \neq f$. Following the isoparametric concept, a referential or parametric element $\overline{\mathscr{T}}$ is defined, and takes the role of the parametric body $\bar{\Omega}$. The parametric element is mapped into each undeformed element $\mathscr{T}_{0}^{e}$ through the elemental undeformed configuration $\varphi_{0}^{e}$; thus, the finite element undeformed configuration $\varphi_{0}^{h}$ is a parametrization of $\Omega_{0}$ defined element-wise. It can be written in terms of the shape functions $N_{I}\left(\xi^{1}, \xi^{2}\right)$ and nodal coefficients. For each of the components $A$ of the discrete undeformed configuration $\varphi_{0}^{h}$ in the co-ordinate system $\left\{X^{1}, X^{2}\right\}$, and for each element $e$ we have:

$$
\left(\varphi_{0}^{e}\right)^{A}\left(\xi^{1}, \xi^{2}\right)=\sum_{I}\left(\varphi_{0}^{e}\right)_{I}^{A} N_{I}\left(\xi^{1}, \xi^{2}\right), \quad \forall\left(\xi^{1}, \xi^{2}\right) \in \overline{\mathscr{T}}
$$

where $\left(\varphi_{0}^{e}\right)_{I}^{A}$ are the nodal coefficients in the local element numbering, while according to the above mentioned notation, the nodal coefficients in the global numbering are $\left(\varphi_{0}^{h}\right)_{J}^{A}$.

The finite element deformed configuration $\varphi^{h}$ is defined in a similar fashion. Each element, $\overline{\mathscr{T}}$ is mapped into the deformed element $\mathscr{T}^{e}$ through $\varphi^{e}$. The finite element approximation of the position vectors is

$$
\boldsymbol{\varphi}^{e}\left(\xi^{1}, \xi^{2}\right)=\sum_{I} \boldsymbol{\varphi}_{I}^{e} N_{I}\left(\xi^{1}, \xi^{2}\right), \quad \forall\left(\xi^{1}, \xi^{2}\right) \in \overline{\mathscr{T}}
$$




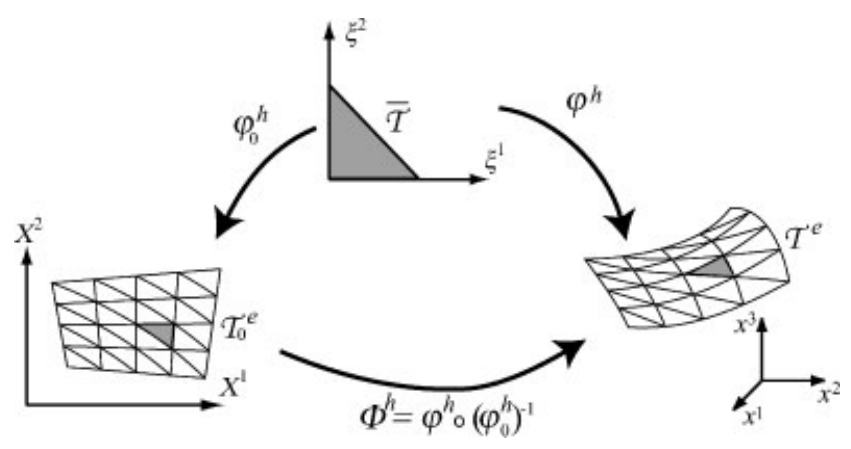

Figure 4. Finite element discretization.

The nodal degrees of freedom of the system are $\boldsymbol{\varphi}_{J}^{h}$, while $\left(\varphi_{0}^{h}\right)_{J}$ define the undeformed configuration. Note that the vector nodal coefficients $\boldsymbol{\varphi}_{J}^{h}$ only corresponds to the position vector of node $J$ if the finite element scheme is an interpolation; the one used here is not. The finite element deformation map is then $\Phi^{h}=\varphi^{h} \circ\left(\varphi_{0}^{h}\right)^{-1}$. This general setting is sketched in Figure 4.

Here, subdivision finite elements based on Loop's scheme are used [58]. There are 12 shape functions $N_{I}\left(\xi^{1}, \xi^{2}\right)$ (the quartic box spline shape functions); the approximated field within a triangular element depends not only on the nodal coefficients of its three nodes, but also on the coefficients of its first neighborhood of nodes. This approximation scheme produces $H^{2}$ fields, i.e. fields with up to second square integrable derivatives. This is crucial in the present theory since the strain energy density depends the curvature of the surface $\mathscr{K}$, which therefore needs to be square integrable. The need of finite second derivatives of the shape functions is clear from Equation (47) below. Any other smooth enough discrete parametrization of the surface can be used instead of subdivision finite elements, e.g. the element-free Galerkin approximation [59].

4.1.2. Calculation of the strain measures. The convected basis vectors can be computed, recalling Equations (2) and (45), for each element $e$ as

$$
\mathbf{g}_{\alpha}=\sum_{I} N_{I, \alpha} \boldsymbol{\varphi}_{I}^{e}, \quad \text { or } \quad\left(g_{\alpha}\right)^{a}=\sum_{I}\left(\varphi^{e}\right)_{I}^{a} N_{I, \alpha}
$$

Note that these vectors are tangent to the finite element surface $\mathscr{T}^{e}$. For the curvature tensor, the derivatives of the convected basis vectors are needed, and are obtained as

$$
\mathbf{g}_{\alpha, \beta}=\sum_{I} N_{I, \alpha \beta} \boldsymbol{\varphi}_{I}^{e}, \quad \text { or } \quad\left(g_{\alpha, \beta}\right)^{a}=\sum_{I}\left(\varphi^{e}\right)_{I}^{a} N_{I, \alpha \beta}
$$

For the pull-back operations (see Equations (9) and (13)), the matrix elements of $\left[T \varphi_{0}^{h}\right]_{\mathscr{B}_{0} \overline{\mathscr{B}}}$ in each element $e$ are

$$
\left(T \varphi_{0}^{e}\right)_{\alpha}^{A}=\frac{\partial\left(\varphi_{0}^{e}\right)^{A}}{\partial \xi^{\alpha}}=\sum_{I}\left(\varphi_{0}^{e}\right)_{I}^{A} N_{I, \alpha}
$$

This $2 \times 2$ matrix needs to be inverted once at each integration point at the beginning of the computation, is then stored and retrieved each time a tensor needs to be pulled-back. 


\subsection{Discrete minimization problem}

Stable configurations of the discrete system are obtained by direct minimization of the potential energy $\Pi\left[\Phi^{h}\right]$ of the discretized system. Numerical methods that only require gradients of the discrete energy with respect to the degrees of freedom have been used, namely the Conjugate Gradients method and the BFGS quasi-Newton method [60,61]. We now describe the numerical calculation of the internal and non-bonded energy, as well as the nodal forces. The external contributions are straightforward.

4.2.1. Internal energy. Since the undeformed configuration $\varphi_{0}^{h}$ is fixed, the finite element deformation map $\Phi^{h}$ is determined by the nodal coefficients $\boldsymbol{\varphi}_{J}^{h}$. The internal energy for this deformation map is computed by splitting the integral over the undeformed body into elements, transforming these element integrals to integrals in the referential element, and approximating these integrals by numerical quadrature:

$$
\begin{aligned}
\Pi_{\text {int }}\left[\Phi^{h}\right] & =\int_{\Omega_{0}} \hat{W}\left(\mathbf{C}\left(\Phi^{h}\right), \mathscr{K}\left(\Phi^{h}\right)\right) \mathrm{d} \Omega_{0} \\
& =\sum_{e=1}^{\text {nel }} \int_{\overline{\mathscr{T}}} \hat{W}(\mathbf{C}, \mathscr{K}) \operatorname{det}\left(T \varphi_{0}^{e}\right) \mathrm{d} \overline{\mathscr{T}} \\
& \left.\approx \sum_{e=1}^{\text {nel }} \sum_{i=1}^{\text {nint }} \hat{W}(\mathbf{C}, \mathscr{K})\right|_{\xi_{i}} \underbrace{\left.\operatorname{det}\left[\left(T \varphi_{0}^{e}\right)^{A}{ }_{\alpha}\right]\right|_{\xi_{i}} \bar{\omega}_{i}}_{\omega_{i}^{e}}
\end{aligned}
$$

where nint is the number of quadrature points for the internal energy, $\xi_{i}$ denote the quadrature points and $\bar{\omega}_{i}$ the corresponding weights. The definition of the weights $\omega_{i}^{e}$ for the deformed element, which include the determinants, is convenient for subsequent equations.

4.2.2. Internal forces. The internal nodal forces of the discrete system are the derivatives of the internal energy with respect to the nodal degrees of freedom

$$
\left(\mathbf{f}_{\text {int }}\right)_{J}=\frac{\partial \Pi_{\text {int }}}{\partial \varphi_{J}^{h}}
$$

where $\left(\mathbf{f}_{\text {int }}\right)_{J}$ corresponds to the global numbering. The application of the chain rule allows us to compute the elemental contributions to these forces elementwise:

$$
\begin{aligned}
\left(\mathbf{f}_{\text {int }}^{e}\right)_{I} & =\int_{\overline{\mathscr{T}}}\left(\frac{\partial \hat{W}}{\partial C_{A B}} \frac{\partial C_{A B}}{\partial \boldsymbol{\varphi}_{I}^{e}}+\frac{\partial \hat{W}}{\partial \mathscr{K}_{C D}} \frac{\partial \mathscr{K}_{C D}}{\partial \boldsymbol{\varphi}_{I}^{e}}\right) \operatorname{det}\left(T \varphi_{0}^{e}\right) \mathrm{d} \overline{\mathscr{T}} \\
& \left.\approx \sum_{i=1}^{\operatorname{nint}}\left(\frac{1}{2} S^{A B} \frac{\partial C_{A B}}{\partial \boldsymbol{\varphi}_{I}^{e}}+m^{C D} \frac{\partial \mathscr{K}_{C D}}{\partial \boldsymbol{\varphi}_{I}^{e}}\right)\right|_{\xi_{i}} \omega_{i}^{e}
\end{aligned}
$$

where $\omega_{i}^{e}$ is defined in Equation (49). These elemental forces are then gathered into the global internal force array $\left(\mathbf{f}_{\text {int }}\right)_{J}$. The computation of the internal energy and forces in each element 
Box 2. Calculation of the elemental internal energy and forces.

- Initialize $\Pi_{\text {int }}^{e}=0,\left(f_{\text {int }}^{e}\right)_{I}^{a}=0$.

- Scatter $\left(\varphi^{e}\right)_{I}^{a}$ from $\left(\varphi^{h}\right)_{J}^{a}$.

- Loop over the quadrature points $i=1$, nint (everything evaluated at $\xi_{i}$ ):

1. Compute the Green deformation tensor (following Equations (46), (7), (9) and (48)), and its derivatives with respect to the degrees of freedom (see Appendix B):

$$
C_{A B}, \quad \frac{\partial C_{A B}}{\partial\left(\varphi^{e}\right)_{I}^{a}}
$$

2. Compute the (pull-back of the) curvature tensor (following Equations (11), (47), (12), and (13)), and its derivatives with respect to the degrees of freedom (see Appendix B):

$$
\mathscr{K}_{A B}, \quad \frac{\partial \mathscr{K}_{A B}}{\partial\left(\varphi^{e}\right)_{I}^{a}}
$$

3. Compute the strain energy density $\hat{W}(\mathbf{C}, \mathscr{K})$ and the stress tensors $S^{A B}$ and $m^{A B}$ (see Box 1).

4. Increment the elemental internal energy and forces:

$$
\begin{aligned}
\Pi_{\mathrm{int}}^{e} & \leftarrow \Pi_{\mathrm{int}}^{e}+\hat{W} \omega_{i}^{e}, \\
\left(f_{\mathrm{int}}^{e}\right)_{I}^{a} & \leftarrow\left(f_{\mathrm{int}}^{e}\right)_{I}^{a}+\left(\frac{1}{2} S^{A B} \frac{\partial C_{A B}}{\partial\left(\varphi^{e}\right)_{I}^{a}}+m^{C D} \frac{\partial \mathscr{K}_{C D}}{\partial\left(\varphi^{e}\right)_{I}^{a}}\right) \omega_{i}^{e}
\end{aligned}
$$

- Gather $\left(f_{\text {int }}^{e}\right)_{I}^{a}$ into $\left(f_{\text {int }}^{h}\right)_{J}^{a}$, and add $\Pi_{\text {int }}^{e}$ to the total internal energy

is summarized in Box 2. The details of the calculation of the derivatives of the strain measures with respect to the nodal degrees of freedom are provided in Appendix B. It is worth mentioning that the symmetry of the strain and the stress tensors is taken advantage of in the computer implementation by means of Voigt notation [57].

4.2.3. Non-bonded energy. It is important to omit the non-bonded energy of pairs of integration points which, in the undeformed body, lie within the bonding distance. However, for the sake of simplicity, this fact is not noted in the following expressions (see Equation (36)). The numerical evaluation of the non-bonded energy entails a double integral, which is performed as a double loop over the elements. Because of the high computational cost of this operation, the computer implementation includes a binning algorithm to search for close (within van der Waals interaction distance) neighbors, and the neighbor lists are updated every few energy evaluations. The numerical approximation of this energy can be written as

$$
\Pi_{\mathrm{nb}}\left[\Phi^{h}\right]=\frac{1}{2} \int_{\Omega_{0}} \int_{\Omega_{0}-B_{X}} \mathscr{V}_{\mathrm{nb}}\left(\left\|\boldsymbol{\Phi}^{h}(X)-\boldsymbol{\Phi}^{h}(Y)\right\|\right) \mathrm{d} \Omega_{0 Y} \mathrm{~d} \Omega_{0 X}
$$




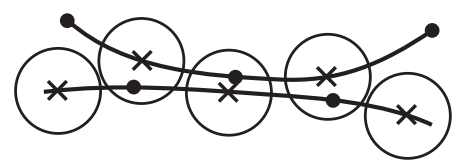

Figure 5. Two numerical surfaces coming to van der Waals contact, but failing to feel it because of insufficient quadrature points: the finite element nodes are represented by $\bullet$, the quadrature points for the non-bonded term by $X$, and the van der Waals cut-off radius by circles, which here do not overlap.

$$
\begin{aligned}
& =\sum_{e=1}^{\mathrm{nel}} \int_{\overline{\mathscr{T}}} \sum_{f=e+1}^{\mathrm{nel}} \int_{\overline{\mathscr{T}}} \mathscr{V}_{\mathrm{nb}}\left(\left\|\boldsymbol{\varphi}^{e}(\xi)-\boldsymbol{\varphi}^{f}(\zeta)\right\|\right) \operatorname{det}\left(T \varphi_{0}^{e}\right) \operatorname{det}\left(T \varphi_{0}^{f}\right) \mathrm{d} \overline{\mathscr{T}}_{\zeta} \mathrm{d} \overline{\mathscr{T}}_{\xi} \\
& =\sum_{e=1}^{\mathrm{nel}} \sum_{i=1}^{\mathrm{nnb}} \sum_{f=e+1}^{\mathrm{nel}} \sum_{j=1}^{\mathrm{nnb}} \mathscr{V}_{\mathrm{nb}}\left(\|\underbrace{\boldsymbol{\varphi}^{e}\left(\xi_{i}\right)-\boldsymbol{\varphi}^{f}\left(\zeta_{j}\right)}_{\boldsymbol{\varphi}_{i-j}^{e-f}}\|\right) \omega_{i}^{e} \omega_{j}^{f}
\end{aligned}
$$

where $\boldsymbol{\varphi}_{i-j}^{e-f}$ denotes the vector defined by the $i$ th quadrature point of element $e$ and the $j$ th quadrature point of element $f$, nnb is the number of quadrature points for the nonbonded term, and $\omega_{i}^{e}$ is defined in Equation (49). Note that the numerical quadrature for the integration of the non-bonded term need not be the same as that for the internal energy, i.e. in general $\mathrm{nnb} \neq$ nint. The latter is obviously independent of the element size, while the former is determined by the van der Waals cut-off distance relative to the element size. The non-bonded potential decays with distance, and a cut-off radius is usually implemented. Large nanotubes display smooth deformations, which can be accurately represented with very large finite elements relative to this cut-off radius. In this situation, it may be necessary to sample the above integrals with a large number of quadrature points to accurately resolve the scale of the non-bonded interactions. Otherwise, two very close surfaces could fail to 'feel' the van der Waals interactions simply because the quadrature points sampling the above integrals on each surface are separated too much (see Figure 5 for an illustration). In the numerical simulations presented later, up to 12 Gauss points per element are needed for the integration of the non-bonded term for the largest nanotubes. Note however that the number of integration points for the non-bonded term is generally considerably smaller than the number of nuclei in the atomistic model, resulting in important computational savings in the calculation of the non-bonded interactions.

4.2.4. Non-bonded forces. We can define a non-bonded energy between elements $e$ and $f>e$ as

$$
\Pi_{\mathrm{nb}}^{e-f}=\sum_{i=1}^{\mathrm{nnb}} \sum_{j=1}^{\mathrm{nnb}} \mathscr{V}_{\mathrm{nb}}\left(\left\|\boldsymbol{\varphi}_{i-j}^{e-f}\right\|\right) \omega_{i}^{e} \omega_{j}^{f}
$$

This energy results in an elemental force in the nodes of element $e$

$$
\left(\mathbf{f}_{\mathrm{nb}}^{e / f}\right)_{I}=\frac{\partial \Pi_{\mathrm{nb}}^{e-f}}{\partial \boldsymbol{\varphi}_{I}^{e}}=\sum_{i=1}^{\mathrm{nnb}} \sum_{j=1}^{\mathrm{nnb}} \frac{1}{\left\|\boldsymbol{\varphi}_{i-j}^{e-f}\right\|} \mathscr{V}_{\mathrm{nb}}^{\prime}\left(\left\|\boldsymbol{\varphi}_{i-j}^{e-f}\right\|\right) N_{I}\left(\xi_{i}\right) \omega_{i}^{e} \omega_{j}^{f} \boldsymbol{\varphi}_{i-j}^{e-f}
$$


as well as a corresponding elemental contribution of the force in element $f$

$$
\left(\mathbf{f}_{\mathrm{nb}}^{f / e}\right)_{I}=\frac{\partial \Pi_{\mathrm{nb}}^{e-f}}{\partial \boldsymbol{\varphi}_{I}^{f}}=-\sum_{i=1}^{\mathrm{nnb}} \sum_{j=1}^{\mathrm{nnb}} \frac{1}{\left\|\boldsymbol{\varphi}_{i-j}^{e-f}\right\|} \mathscr{V}_{\mathrm{nb}}^{\prime}\left(\left\|\boldsymbol{\varphi}_{i-j}^{e-f}\right\|\right) N_{I}\left(\zeta_{j}\right) \omega_{i}^{e} \omega_{j}^{f} \boldsymbol{\varphi}_{i-j}^{e-f}
$$

which are gathered into $\left(\mathbf{f}_{\mathrm{nb}}\right)_{J}$ accordingly.

\section{NUMERICAL VALIDATION OF THE THEORY}

This section describes a series of calculations performed on carbon nanotubes, which are an example of curved crystalline sheets for which many experimental studies have recently become available. In these simulations, nanotubes are deformed incrementally beyond the load where structural instabilities occur, in the full non-linear regime. The positions of the nodes at the end of the tubes are incrementally displaced and, in each step, the energy is minimized. In order to test the accuracy and performance of the continuum/finite element computational scheme, full atomistic calculations for the same nanotubes and loadings are performed independently, and the equilibrium configurations and energies provided by the two methods are compared. We emphasize that the continuum model does not come from fitting material parameters to match atomistic simulations, but rather a given model for the interatomic potential is used both in the atomistic calculations and to construct the continuum model. The continuum model is thus intended to mimic the atomistic model, which is assumed to be 'true'. Therefore, when we speak of the accuracy or the error of the continuum model, the reader should understand that the atomistic model at hand is taken as the reference. In the following examples, the Tersoff-Brenner potential is adopted (the second parameter set in Reference [53]) for the bonded interactions, and the Lennard-Jones potential for the nonbonded interactions corresponds to the graphene-graphene parameter set in Reference [56].

As mentioned before, subdivision finite elements based on Loop's scheme [58] have been used. In this method, a control surface mesh whose nodes have only translational degrees of freedom parameterizes the surface. The control or computational mesh is presented in one of the examples below as a faceted surface. In most cases, the post-processed smooth surface defined by the computational mesh is shown. This smooth surface is the actual numerical representation of the deformation of the continuum membrane, while the computational mesh only represents the degrees of freedom of the discrete model.

The molecular mechanics and the finite element computer codes are comparable, and actually share many routines like those defining the interatomic potentials and the minimization routine. Therefore, the computational times can be used to compare the two methods. For a given number of degrees of freedom, the calculation of the energy and the forces for the continuum/finite element model is more expensive than for the atomistic system (the metric and the curvature tensors of the surface, as well as their derivatives with respect to the nodal degrees of freedom must be computed, and the inner relaxation must be performed). Nevertheless, the reduction of degrees of freedom that the continuum model allows for a given problem makes this approach much more efficient when many atoms correspond to an element. This is not only due to the computational savings in each calculation of the energy and the forces for a much smaller system, but also due to the reduction in degrees of freedom of the continuum/finite element systems that speeds the convergence of the minimization routine. 

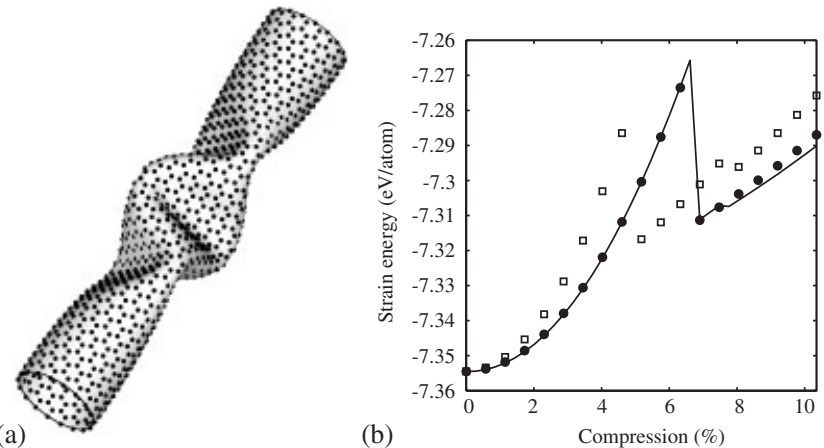

Figure 6. Compressed $8.7 \mathrm{~nm}$ long $(18,0)$ nanotube, comparison between the full atomistic model and the continuum/finite element model: (a) super-imposed deformed configurations for atomistic (black spheres) and finite element (grey surface) calculations; and (b) strain energy evolution for the atomistic model $(-)$ and for the continuum simulation $(\bullet)$. The strain energy for a continuum model in which the inner displacements are not relaxed is also depicted $(\square)$.

Furthermore, it is possible to take larger load increments with the continuum/finite element model without compromising the convergence of the minimization routine - in the computational time comparisons, however, the number of load steps is the same for the atomistic and the continuum calculations.

\subsection{Compressed $(18,0)$ nanotube}

Figure 6 shows the results for a $(18,0)$ zig-zag nanotube compressed beyond the buckling point. In this example, both the atomistic and the finite element model have about 4300 degrees of freedom. The superimposed final deformed configurations for the continuum/finite element and atomistic calculations are reported for a compression of $10.35 \%$. The characteristic system of 'fins' described in the literature [1], i.e. perpendicular flattenings of the tube, are clearly visible. The morphological agreement between the continuum and the atomistic simulations is remarkable, and the deformed membrane nearly coincides exactly with the positions of the nuclei provided by the atomistic calculation. Figure 6(b) presents the evolution of the internal or binding energy of the system as a function of the compression. For reference, the TersoffBrenner potential predicts a ground energy for graphene of $E_{\text {graphene }}=-7.3756 \mathrm{eV} /$ atom. Before the buckles form, the energy displays a nearly quadratic growth. In this stage, the energies provided by the two methods are undistinguishable (comparable to the minimization tolerance). Note that the energy evolution is matched in absolute terms; for the comparison, neither of the curves has been shifted vertically. The buckling load is correctly predicted by the continuum simulation. After buckling, the energy grows nearly linearly. In this regime, characterized by very large local deformations, the continuum approach is also accurate; the error in the last reported step is $4 \%$ (this percentage, as all results reported subsequently, is relative to the strain energy variation, i.e. energy at the observation point minus the initial energy).

This plot also reports the evolution of the strain energy for the continuum model, without the inner displacement relaxation, in order to illustrate its crucial role in the correct modelling of the elasticity of nanotubes. It can be observed that the errors are considerable; before buckling occurs, the strain energy is over-estimated by $60 \%$, and at the end by about $20 \%$. 


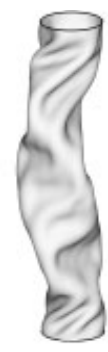

(a)

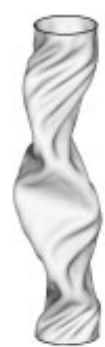

(b)

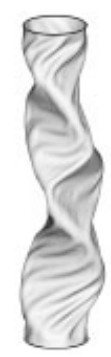

(c)

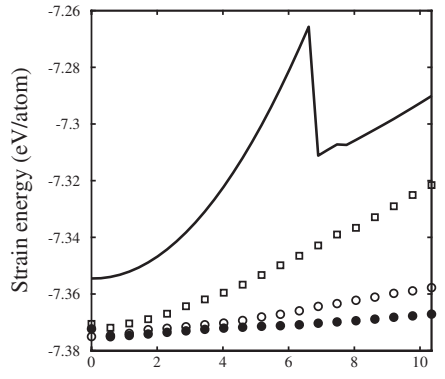

(d)

Compression $(\%)$

Figure 7. Compressed $8.7 \mathrm{~nm}$ long $(18,0)$ nanotube: unphysical deformations obtained with the standard Cauchy-Born rule for coarse (a), regular (b), and fine (c) meshes, (d) strain energy evolution for the atomistic model (-) and for the continuum simulation based on the standard Cauchy-Born rule for the coarse $(\square)$, the regular $(\circ)$, and the fine $(\bullet)$ meshes.

The buckling point is severely under-estimated in the absence of the internal relaxation. In some cases, even the deformation modes are not correctly predicted without the internal relaxation.

This simulation provides a stringent test for the continuum theory since it concerns a very small nanotube, only a few atoms in diameter, which is severely deformed with local radii of curvature that approach the bond length. From a practical point of view, the continuum formulation is aimed at larger or longer nanotubes, for which it provides significant computational savings, as illustrated later. Nevertheless, an attractive attribute of this model is its good performance even for such severe deformations, even when the scale of the finite elements is comparable to the scale of the bond lengths.

\subsection{Results with the standard Cauchy-Born rule}

This excellent behaviour contrasts with a continuum membrane model directly constructed from the standard Cauchy-Born rule without the exponential extension given in Reference [44]. As discussed in Reference [44], the energy of a membrane model based on the standard Cauchy-Born rule is invariant under isometric deformations (deformations that keep $\mathbf{C}$ unchanged, i.e. bending without stretch). Thus, this energy does not depend on the curvature of the membrane, and the model has zero bending stiffness. This non-convexity of the hyperelastic potential manifests itself as a pathological mesh dependency. Because of the finite dimension of the discrete FE space, and fact that the boundary conditions may not be compatible with an isometric deformation, the numerical method still finds a solution which minimizes the total discrete energy $\Pi\left[\Phi^{h}\right]$. Nevertheless, as the mesh is refined, the numerical method selects solutions with increasingly finer features.

Figure 7 illustrates this behaviour for the compressed nanotube of the previous example. The results are provided for three meshes, consisting of 820, 1830, and 3240 nodes. As the mesh is refined, the numerical solution is able to develop finer folds, and accommodate the deformation nearly isometrically. These solutions do not correspond to the behaviour of the compressed carbon nanotube depicted in Figure 6. This mesh dependency can also be observed in the energy evolution. It can be observed that as the mesh is refined, the increase of energy during the deformation almost vanishes. The energy evolution for the atomistic 

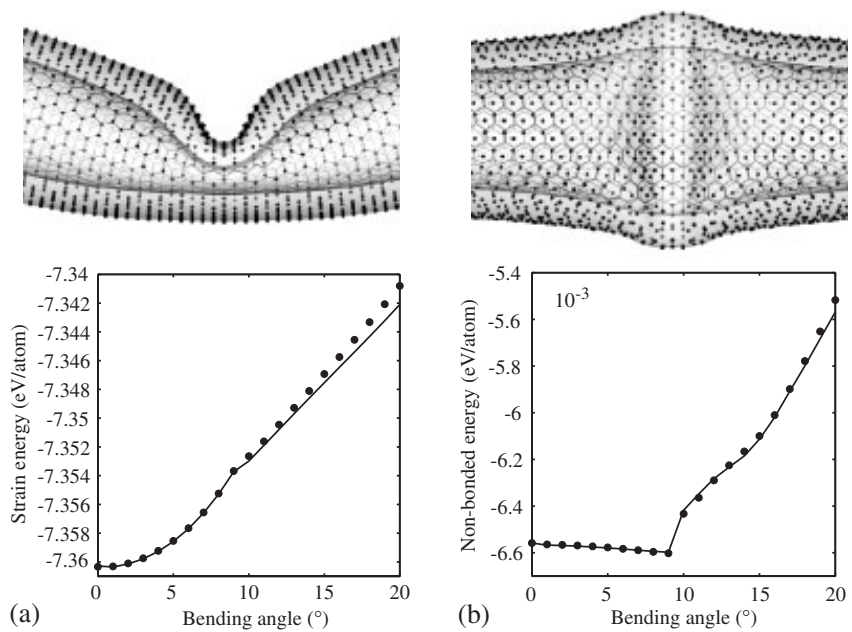

Figure 8. Bent $12.56 \mathrm{~nm}$ long $(10,10),(15,15)$ two-walled nanotube: (a) superimposed deformed configurations for atomistic (black spheres, and solid lines) and finite element (translucent grey surface) calculations; and (b) strain and non-bonded energy evolution for the atomistic model $(-)$ and for the continuum simulation $(\bullet)$.

system is provided to highlight these unphysical results. These phenomena are reminiscent of the response of materials for which the strain energy density is physically non-convex (e.g. martensitic materials or nematic elastomers), which develop microstructures with increasingly fine features in the process of energy minimization $[62,63]$.

\subsection{Bent $(10,10),(15,15)$ two-walled nanotube}

Next, we describe an example in which the non-bonded interactions are critical. A twowalled nanotube is bent by rotating each end by $20^{\circ}$ in opposite directions with respect to an axis perpendicular to the axis of the undeformed nanotube, and passing through its centre. At an angle near $9^{\circ}$, a single buckle forms in the centre of the two-walled nanotube. The nanotubes are chosen so that initially, their walls are at approximately the van der Waals equilibrium distance. Figure 8 reports the deformed configurations for the atomistic and the continuum/finite element models at the end of the simulation, with a side and a top view of the buckle. To facilitate the visualization, the continuum solution is displayed as a translucent grey surface. The atomistic model is displayed by black spheres for the outer tube and solid lines for the bonds of the inner tube. It can be observed that the outer tube displays a sharper kink than the inner tube, in agreement with reported experimental observations and atomistic calculations [8]. Again, the agreement between the two models, despite the very large, localized deformations, is remarkable. The evolution of the deformation energy is also very well predicted, again in absolute terms, with perfect matching in the quadratic regime, and only slight discrepancies later. At the final stage, the error is about $6 \%$. The evolution of the non-bonded energy as a function of the bending angle is also provided, and the agreement is excellent, which demonstrates the accuracy of the continuum version of these interactions. The buckling angle can be identified in this plot as a sharp increase of the non-bonded energy, probably due to the compression between the walls of the nanotubes at the buckle. 


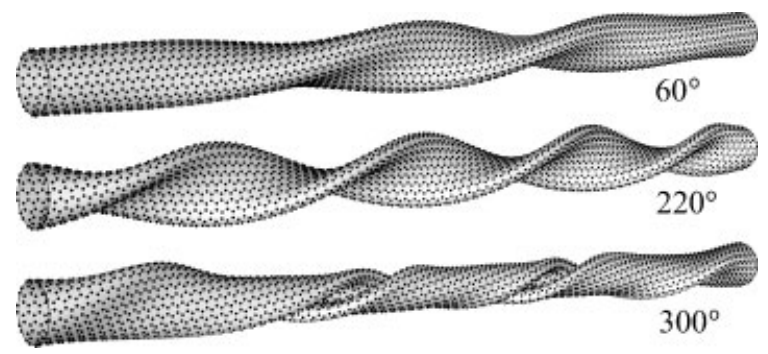

Figure 9. Twisted $25.11 \mathrm{~nm}$ long $(10,10)$ nanotube: super-imposed deformed configurations at three twisting angles for atomistic calculation (black spheres) and continuum finite element calculation (grey surface).
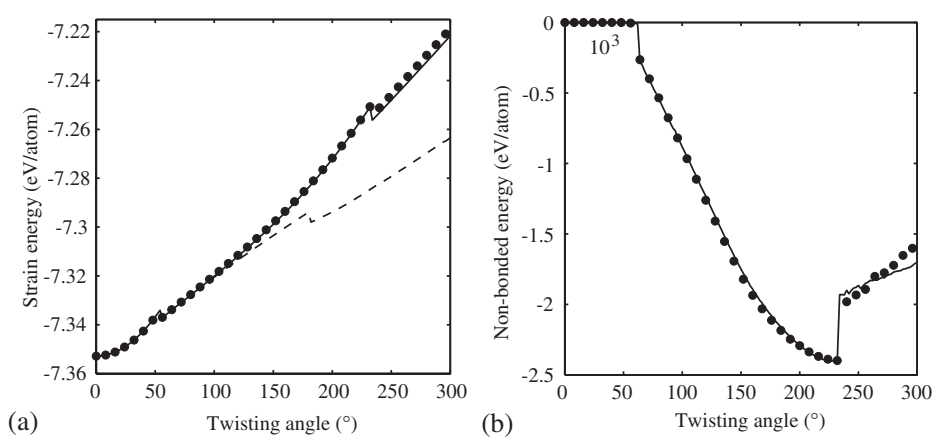

Figure 10. Twisted $25.11 \mathrm{~nm}$ long $(10,10)$ nanotube: (a) comparison of the strain energy as a function of the twisting angle for atomistic calculation (-), and continuum/FE calculation $(\bullet)$, and strain energy density evolution if the non-bonded interactions are ignored (- - ); and (b) comparison of the non-bonded energy evolution for atomistic calculation $(-)$ and continuum/FE calculation $(\bullet)$.

Note that the change of non-bonded energy is much smaller than the change of strain energy. Nevertheless, the non-bonded interactions determine the morphology of the buckle, and interpenetration of the walls of the two nanotubes will occur in their absence. In this example, both models have around 15000 degrees of freedom, and the computation time is two times longer for the continuum/finite element approach. The objective of this example is not to demonstrate the computational savings that the continuum model can provide, but rather to show its accuracy for highly strained multi-walled nanotubes.

\subsection{Twisted $(10,10)$ nanotube}

In this example, a $(10,10)$ nanotube $25 \mathrm{~nm}$ long is twisted by rotating its ends in opposite directions with respect to the axis of the tube. Three representative snapshots of the deformation process are shown in Figure 9. The evolution of the strain energy is presented for both the atomistic and the continuum/finite element calculations in Figure 10. The strain energy evolution if the non-bonded interactions are not included is also reported in this figure (dashed line). The evolution of the non-bonded energy is also presented. This example exhibits two structural instabilities. In the first one, a non-uniform deformation mode develops for 
Table I. Twisted $25.11 \mathrm{~nm}$ long $(10,10)$ nanotube: error of the four considered finite element models at two twisting angles.

\begin{tabular}{lcccc}
\hline & $\begin{array}{c}\text { Coarse } \\
(\%)\end{array}$ & $\begin{array}{c}\text { Medium } \\
(\%)\end{array}$ & $\begin{array}{c}\text { Fine } \\
(\%)\end{array}$ & $\begin{array}{c}\text { Super fine } \\
(\%)\end{array}$ \\
\hline $220^{\circ}$ & 1.1 & 0.39 & 0.37 & 0.29 \\
$300^{\circ}$ & 7.4 & 3.6 & 2.3 & 1.5 \\
\hline
\end{tabular}

a twisting angle at each end of about $50^{\circ}$. The onset of this instability is evident in the first snapshot of the deformation in Figure 9, and can be identified in the strain energy evolution as the kink that ends the nearly quadratic regime. As loading proceeds, the wall of the tube comes into van der Waals contact with itself and adhesion energy is gained. Then, the van der Waals interactions harden the twisting response of the tube. This can be noticed by observing the deviation between the response with (solid) and without (dashed) non-bonded interactions.

The dashed line demonstrates the fundamental effect on the global response of these interactions, despite being less than $2 \%$ of the total energy change. The second kink in the strain energy evolution, near $230^{\circ}$, indicates the development of a secondary structure. After this point, the flattened twisted ribbon folds onto itself. The snapshots demonstrate that, even for these intricate deformed morphologies, the continuum mechanics theory is surprisingly accurate, and the finite element model remarkably fits the atomic positions. When it comes to the energetics, the agreement is also excellent, both for the strain and the non-bonded energies (note that the discrepancy in the non-bonded interactions at $300^{\circ}$ is only $0.1 \%$ of the total energy variation).

This analysis has been performed with four different meshes, a coarse one with 6666 degrees of freedom and 22 elements in the perimeter, a medium one with 10164 degrees of freedom and 28 elements in the perimeter, a fine one with 16520 degrees of freedom and 32 elements in the perimeter, and a super-fine mesh with over 30000 degrees of freedom. The atomistic system has 12000 degrees of freedom. The results reported above are for the medium mesh. The relative errors of the finite element models before the second instability $\left(220^{\circ}\right)$ and at the end of the analysis $\left(300^{\circ}\right)$ are reported in Table I. Note that this error includes both the finite element approximation error, and the continuum modelling error. The table shows that the finite element solution at $220^{\circ}$ is excellent, even for the coarse mesh. After the second instability, the severe deformation makes the analysis with the coarse mesh less accurate. It is observed that mesh refinement reduces the errors.

This simulation suggests that local deformation features do not necessarily require coupling with atomistic calculations, and simple mesh refinement is sufficient to obtain accurate solutions, as indicated in the table. Of course, from a practical point of view, it makes little sense to model an atomistic system with an approximate finite element model with more degrees of freedom. As shown in the next example, accurate computations of larger tubes, which typically display deformation features that are larger relative to the bond length, require finite element models with far fewer degrees of freedom than the atomistic model.

\subsection{Twisted $(30,30)$ nanotube}

In example, a larger $(30,30)$ nanotube is twisted until the tube flattens and folds onto itself. Figure 11 shows the superimposed deformations for the atomistic and the continuum/finite 

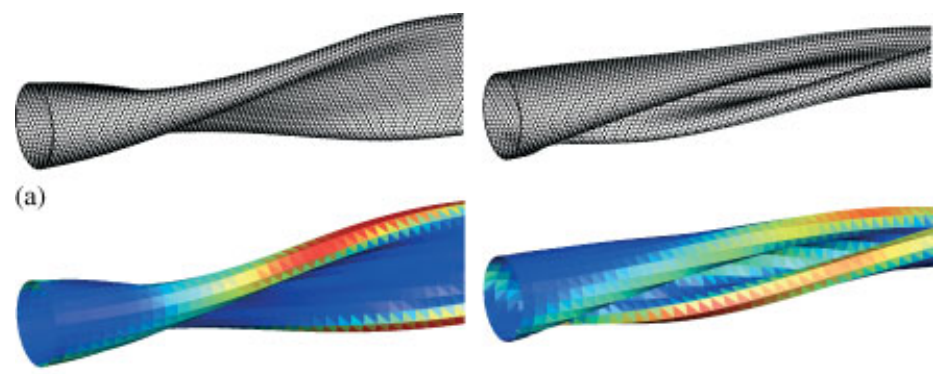

(b) $50^{\circ}$

$75^{\circ}$

Figure 11. Twisted $37.67 \mathrm{~nm}$ long (30,30) nanotube: comparison between the atomistic model and the continuum/finite element model for two twisting angles: (a) super-imposed deformed configurations for atomistic (black spheres) and finite element (grey surface) calculations; and (b) map of the strain energy density on the finite element computational mesh (red is high, blue is low).

element calculations at two twisting angles. As before, the atoms coincide with the continuum membrane. The map of the strain energy density on the computational finite element mesh is also shown. It can be observed that the severely bent areas display high strain energy. In this case, the atomistic system has 54000 degrees of freedom while the continuum/finite element model only 5070. The computational time with the continuum/finite element approach is seven times smaller than with the full atomistic calculation. This fast finite element calculation provides an accurate solution within $0.8 \%$ in strain energy for the first reported twisting angle, and within 5\% for the final angle. Again, a finite element model with a finer mesh of 9696 degrees of freedom (three times faster than the atomistic simulation) reduces the error at $75^{\circ}$ below $1.3 \%$.

\section{LARGE-SCALE EXAMPLES}

This section describes some examples of the bending of multi-walled carbon nanotubes (nested tubes in van der Waals contact). In these examples consisting of up to millions of atoms, the finite element model benefits from the fact that the element sizes can be chosen irrespective of the atomic spacing. Consequently, the computational cost is greatly reduced. These calculations are performed with a parallel implementation of the method. The parallelization is performed on the calculation of the energy and the forces, since most of the CPU time is spent in these operations, and not in the minimization algorithm. For the larger examples of Section 6.2, up to 20 processors have been used at $80 \%$ efficiency.

\subsection{Bent 5-walled nanotube}

There have been numerous experimental observations of bent CNTs displaying distinct kinks or localized buckles (see Section 5.3). For example in Reference [8], a series of TEM (transmission electron microscope) images of bent and buckled single and multi-walled carbon nanotubes were presented. These experiments showed bent 5-walled nanotubes displaying single kinks, as well as patterns of double kinks. We present a series of calculations which predict 
not only the one and two kink patterns, but also a three kink pattern. Systems of consecutive kinks in multi-walled hollow carbon nanotubes have been reported in Reference [64].

In the continuum/finite element calculations, a 5-walled $(23,23)(28,28)(33,33)(38,38)$ $(43,43)$ multi-walled nanotube whose dimensions match those of the experiments is considered. The corresponding atomistic system for a $35 \mathrm{~nm}$ long nanotube has about 276000 degrees of freedom, while the finite element model has 20000.

For this larger nanotube, the first kink forms when both ends of the nanotube are rotated only $5^{\circ}$. For moderate bending angles, until $18^{\circ}$, this is the only deformation mode observed. For larger bending angles, depending on slight perturbations of computational parameters such as the number of load steps or the minimization tolerances, two equilibrium paths can be distinguished. A series of calculations can be qualitatively classified as following path A, or path B (see Figure 12). Path A is characterized by a system of three buckles, while path B displays only two. The figure shows three-dimensional images of the numerical deformations, as well as longitudinal sections. The sections are particularly useful since they reveal the internal structure of the deformation, and they are the numerical analogs of the experimental TEM slices. Cross-sections are also provided for the last configuration of each path, and are marked by thick lines in the longitudinal sections.

These simulations exemplify a feature of large multi-walled CNTs which we expect also in other crystalline sheets: the existence of multiple equilibrium configurations with very different shapes, which nevertheless are nearly indistinguishable from the energetic point of view. Here, path B is slightly energetically favorable. Another characteristic feature of larger CNTs is that they rarely display sharp transitions in the energy evolution; the characteristic quadratic-linear sudden transition observed for the energy evolution of smaller nanotubes in the previous sections is not present here. Indeed, while for unbuckled structures the energy growth with deformation is roughly quadratic, the behaviour of the buckled structures is strongly constrained by van der Waals interactions. This constraint on the kinks causes a stiffening effect clearly observed in the energy evolution.

In addition to the energetics, the simulations also provide a three-dimensional picture of the deformation, unlike TEM which provides 'slices'. This makes the interpretation of experimental observations much easier and complete. For instance, atomistic simulations of bending found in the literature typically involve small nanotubes, which develop kinks with a simple structure [8]. See also Section 5.3 for an illustration. As can be observed in Figure 12 from the longitudinal section for $18^{\circ}$, one may be led to think that the 5-walled nanotube displays the same simple structure. However, the three-dimensional picture reveals two other systems of pairs of buckles tilted in the transverse direction. Actually, the simulation shows that between two simple buckles there is always a pair of tilted buckles, and vice versa. The transverse structure of these types of buckles can observed in the cross-sections of the final configurations. These cross sections also show that the inner-wall delamination, which can be observed both in the experimental and the numerical slices, can be associated with transverse buckles. This alternation of simple centered buckles and pairs of tilted buckles is even more apparent in the following example.

\subsection{Thirty four-walled nanotube}

We next present calculations of a 34-walled $124 \mathrm{~nm}$ long MWCNT containing six million atoms. A 'converged' finite element analysis requires 100000 nodes. The 'rippling' effect 

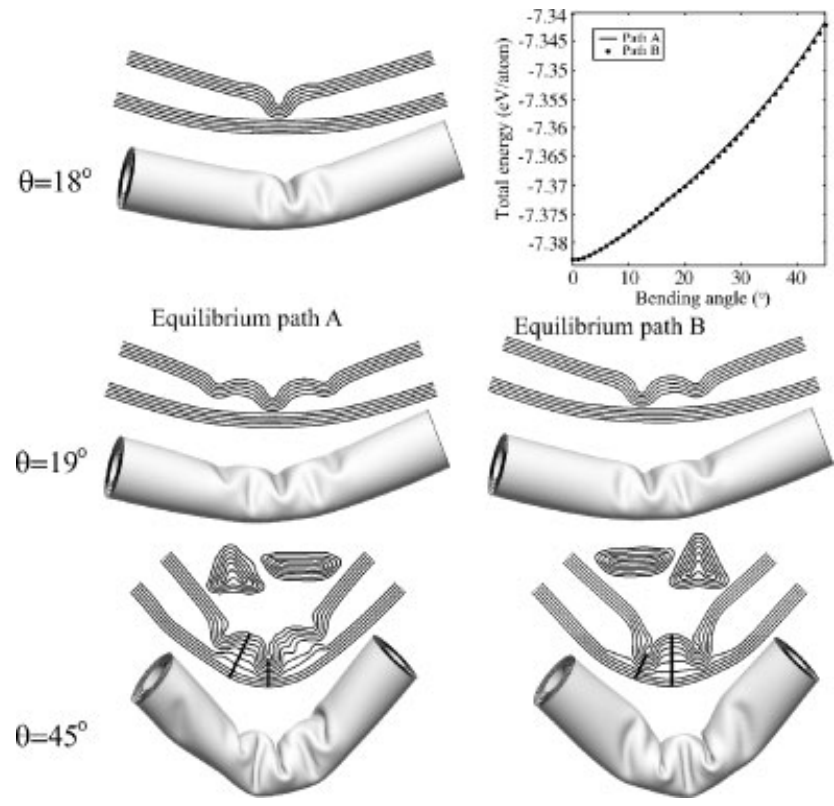

Figure 12. Bending of a 5-walled carbon nanotube, inspired in the experiments by Iijima et al. [8].

observed in experiments $[65,66]$ is reproduced as shown in Figure 13. This nanotube is similar to that reported in Reference [65]. The numerical results show good agreement, for instance in the periodicity of the ripples, although the experimental image seems to indicate that the nanotube is not uniformly bent. The images reported in Reference [66] display smoother ripples, similar to these in our simulations. As before, the calculations provide also the threedimensional picture (see Figure 14), and reveal a feature not apparent from the experimental TEM images: the deformation is a complex pattern of intercalated buckles in tilted orientations, reminiscent of the Yoshimura pattern [67]. This phenomenon, also predicted in torsion, has implications in the analysis and design of nanoevices. See Reference [24] for further details about the non-linear mechanical response and rippling of thick MWCNTs.

\section{SUMMARY AND CONCLUSIONS}

The theory and finite element implementation of a continuum surface model for crystalline monolayers has been presented, and tested against atomistic calculations. The membrane constitutive model is strictly based on the interatomic potential (here the Tersoff-Brenner potential). The theory is strictly framed within continuum mechanics, with a closed-form expression for the elastic potential in terms of continuum strain measures; it does not require constrained atomistic calculations. The last point is of particular importance, since it allows for (semi-)analytical treatments in some situations of interest, as described in References [23,50]. The proposed theory, like other crystal elasticity theories, accounts for the crystallography of the underlying lattice, in particular for the chirality of nanotubes, and treats consistently the 

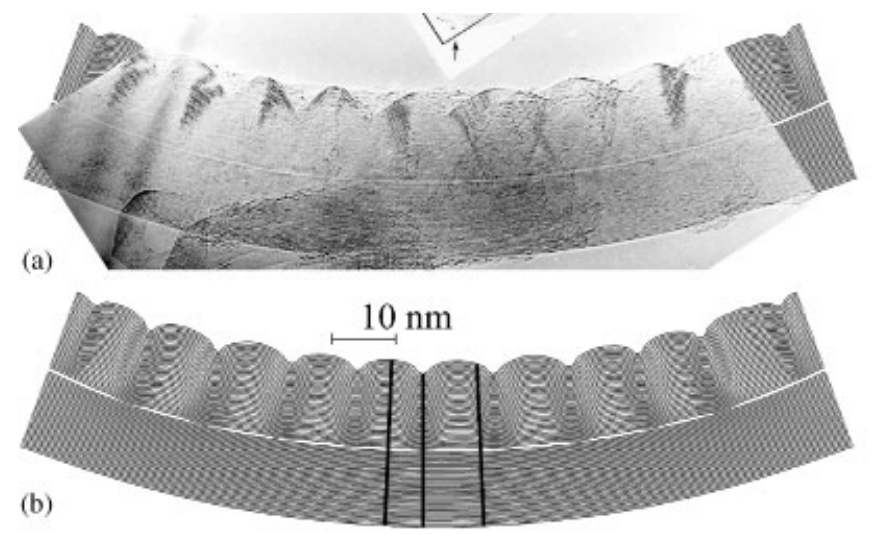

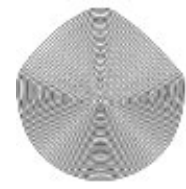

(c)
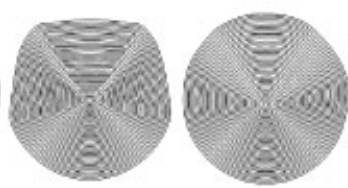

(d)

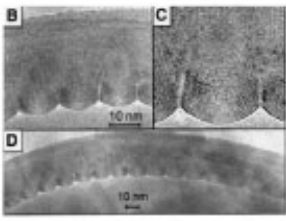

Figure 13. Rippling of a 34-walled carbon nanotube: (a) comparison with experimental observation (reprinted with permission from Kuzumaki et al. [65] Taylor \& Francis Ltd., http://www.tandf.co.uk/journals/titles/14786435.html); (b) side-section; (c) cross-sections of the simulation; and (d) experiment on larger MWCNT (reprinted with permission from Poncharal et al. [66]. Copyright 1999 American Association for the Advancement of Science http://www.sciencemag.org).

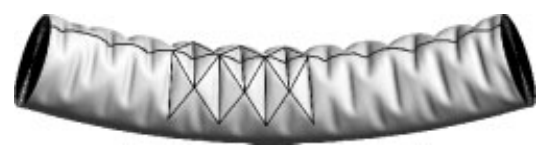

Figure 14. Rippling of a 34-walled carbon nanotube: three-dimensional picture.

inner elasticity of the non-primitive graphene lattice. A continuum version of the non-bonded interactions, crucial in the mechanics at the nanoscale, is provided.

The coarse-grained calculations have been shown to reproduce accurately the full nonlinear mechanics of the parent atomistic system. Our simulations suggest that, in the absence of lattice defects or bond rearrangements, it is possible to accurately describe the non-linear mechanics of carbon nanotubes and other crystalline sheets with exclusively continuum finite element calculations, without any recourse to atomistic calculations. Although this may seem surprising, experimental observations and atomistic simulations of nanotubes in general display smooth deformations (notable exceptions are experimentally observed fracture, and simulated plasticity). These observations can be interpreted as evidence that, as long as the integrity of the lattice is maintained, the mechanical response of carbon nanotubes depends on the atomistic arrangements only through the elasticity of the curved monolayer lattice (the scale of the bond length and that of the overall deformation are well separated). Given the celebrated 
resilience of the carbon network in graphene, our method seems to have a wide range of practical applicability, particularly in the design and analysis of nanotube devices in which only reversible deformations are expected. The finite element calculations allow for accurate simulations at a fraction of the computational cost of conventional atomistic calculations. Note that all of the finite element calculations presented here are performed with uniform meshes. As we have seen, these calculations provide very accurate results for moderate deformations and coarse meshes, but finer meshes are required when the deformations are severe. One of the major advantages of the continuum-based simulations is that the mesh can be tailored to the problem under consideration through adaptive mesh refinement. Furthermore, the mesh can be changed during the analysis very easily since the model is hyperelastic. Thus, the proposed continuum model allows to exploit finite element methods such as adaptivity, which can further reduce the computational cost by orders of magnitude.

Although we have focused on nanoscale applications, these methods are also applicable to lattices on the macroscale. The essential feature of the method is that it relates the deformation of a discrete lattice to that of a membrane with flexural stiffness. If the deformation energy of the discrete system can be expressed in terms of the geometry of the deformed lattice (e.g. the elongation of its elements, the angles between elements), then these methods can be used at any scale.

\section{APPENDIX A: ASPECTS OF THE CONSTITUTIVE MODEL FOR CARBON NANOTUBES}

\section{A.1. Principal curvatures and directions}

The eigenvalues of the generalized eigenvalue problem (15) are the principal curvatures of the surface $\Omega$. They can be easily obtained [48] from the Gaussian curvature

$$
K=\frac{\operatorname{det}[\mathscr{K}]_{\mathscr{B}_{0}}}{\operatorname{det}[\mathbf{C}]_{\mathscr{B}_{0}}}=\frac{\mathscr{K}_{11} \mathscr{K}_{22}-\mathscr{K}_{12}^{2}}{C_{11} C_{22}-C_{12}^{2}}
$$

and the mean curvature

$$
H=\frac{1}{2} \operatorname{trace}\left([\mathbf{C}]_{\mathscr{B}_{0}}^{-1}\left[\mathscr{K}_{\mathscr{B}_{0}}\right)=\frac{1}{2} \frac{\mathscr{K}_{11} C_{22}-2 \mathscr{K}_{12} C_{12}+\mathscr{K}_{22} C_{11}}{C_{11} C_{22}-C_{12}^{2}}\right.
$$

The principal curvatures can then be written as

$$
k_{1,2}=H \pm \sqrt{H^{2}-K}
$$

The two $\mathbf{C}$-orthonormal principal directions $\mathbf{V}_{1}$ and $\mathbf{V}_{2}$ are straight-forward to obtain.

The derivatives of $k_{n}$ and $\mathbf{V}_{n}$ with respect to $\mathbf{C}$ and $\mathscr{K}$ can be obtained from standard formulas [68], valid for the case in which $k_{1} \neq k_{2}$. For the principal curvatures, we have

$$
\frac{\partial k_{n}}{\partial \mathscr{K}}=\mathbf{V}_{n} \otimes \mathbf{V}_{n}, \quad \text { or } \quad \frac{\partial k_{n}}{\partial \mathscr{K}_{A B}}=\left(V_{n}\right)^{A}\left(V_{n}\right)^{B}, \quad n=1,2
$$

and

$$
\frac{\partial k_{n}}{\partial \mathbf{C}}=-k_{n} \frac{\partial k_{n}}{\partial \mathscr{K}}, \quad n=1,2
$$


Introducing the symbol $\otimes_{\text {symm }}$ denoting the symmetrized tensor product, i.e.

$$
\mathbf{A} \otimes_{\text {symm }} \mathbf{B}=\frac{1}{2}(\mathbf{A} \otimes \mathbf{B}+\mathbf{B} \otimes \mathbf{A})
$$

the derivatives of the principal directions can be obtained as

$$
\frac{\partial \mathbf{V}_{n}}{\partial \mathscr{K}}=\frac{1}{\left(k_{n}-k_{m}\right)} \mathbf{V}_{m} \otimes\left(\mathbf{V}_{n} \otimes_{\text {symm }} \mathbf{V}_{m}\right)
$$

or

$$
\frac{\partial\left(V_{n}\right)^{A}}{\partial \mathscr{K}_{B C}}=\frac{1}{2\left(k_{n}-k_{m}\right)}\left(V_{m}\right)^{A}\left[\left(V_{n}\right)^{B}\left(V_{m}\right)^{C}+\left(V_{m}\right)^{B}\left(V_{n}\right)^{C}\right]
$$

and

$$
\frac{\partial \mathbf{V}_{n}}{\partial \mathbf{C}}=-\frac{1}{2} \mathbf{V}_{n} \otimes \mathbf{V}_{n} \otimes \mathbf{V}_{n}-k_{n} \frac{\partial \mathbf{V}_{n}}{\partial \mathscr{K}}
$$

for $n=1,2$ and where $\{n, m\}$ is a permutation of $\{1,2\}$.

For repeated eigenvalues, the procedure for differentiating the eigenvalues and eigenvectors becomes cumbersome [69]. In computations, this rarely occurs, and our experience indicates that numerical differentiation is a simple and robust alternative to compute the stresses at a particular quadrature point for which $\sqrt{H^{2}-K}<$ Tol. Thus, in this case, the derivatives of $k_{n}$ and $\mathbf{V}_{n}$ are not needed.

\section{A.2. Inner relaxation}

In the present Appendix, intrinsic notation is used, although in the computations, all the vectors and tensors are expressed in the Euclidean basis $\mathscr{B}_{0}$. Here, $(\cdot)_{, \boldsymbol{\eta}}$ denotes $\partial(\cdot) / \partial \boldsymbol{\eta}$ and $(\cdot)_{, \eta \eta}$ denotes $\partial^{2}(\cdot) / \partial \boldsymbol{\eta}^{2}$.

A.2.1. Newton's method iterations. The inner relaxation in Equation (29) is performed using Newton's method, by solving at each quadrature point the system of two non-linear algebraic equations which ensure internal equilibrium:

$$
W_{, \boldsymbol{\eta}}=\mathbf{0}
$$

for fixed $\mathbf{C}$ and $\mathscr{K}$. The algorithm can be summarized as

- Initialize $\boldsymbol{\eta}^{0}$ as the last converged inner displacement in the previous energy evaluation

- DO WHILE $\left(\left\|\mathbf{r}^{k}\right\|>\operatorname{Tol}_{1} \cdot\right.$ OR. $\left.\left\|\Delta \boldsymbol{\eta}^{k}\right\|>\operatorname{Tol}_{2}\right)$

$\circ$ Compute the residual $\mathbf{r}^{k}=W_{, \boldsymbol{\eta}}\left(\boldsymbol{\eta}^{k}\right)$

○ Compute the Jacobian matrix $\mathbf{J}^{k}=W_{, \eta \eta}\left(\boldsymbol{\eta}^{k}\right)$

$\circ \Delta \boldsymbol{\eta}^{k}=-\left(\mathbf{J}^{k}\right)^{-1} \mathbf{r}^{k}$

$\circ \boldsymbol{\eta}^{k+1}=\boldsymbol{\eta}^{k}+\Delta \boldsymbol{\eta}^{k}$

$\circ k \leftarrow k+1$

- Check $W\left(\boldsymbol{\eta}^{k}\right)$ is a minimum, i.e. $\operatorname{det} \mathbf{J}^{k}>0$

- $\hat{W}=W\left(\boldsymbol{\eta}^{k}\right)$ and $\hat{\boldsymbol{\eta}}=\boldsymbol{\eta}^{k}$ 
Although no result on the convexity of $W$ as a function of $\boldsymbol{\eta}$ is known to us, numerical experience indicates that for graphene and the Tersoff-Brenner potential, the inner relaxation always converges to a minimum within machine precision in two or three Newton iterations.

A.2.2. Inner forces and inner elastic constants. The residual $\mathbf{r}=W_{, \boldsymbol{\eta}}$ and the Jacobian $\mathbf{J}=W_{\text {, } \boldsymbol{\eta}}$ can be interpreted as inner out-of-balance forces and inner elastic constants [38]. This Section provides details for their calculation. To keep the expressions compact, let us define an array of bond lengths and angles for the three inequivalent bonds of graphene $\mathbf{p}=\left[a_{1}, a_{2}, a_{3}, \theta_{1}, \theta_{2}, \theta_{3}\right]$, and let $p_{i}(i=1,6)$ denote the $i$ th entry of this array.

As for the inner out-of-balance forces, applying the chain rule and recalling Equation (28), we have

$$
W_{, \boldsymbol{\eta}}=\sum_{i=1}^{6} \frac{\partial W}{\partial p_{i}} p_{i, \boldsymbol{\eta}}
$$

On the other hand, the inner elastic constants can be written in the following manner, which highlights the symmetry:

$$
W_{, \boldsymbol{\eta} \eta}=\sum_{i=1}^{6}\left\{\frac{\partial W}{\partial p_{i}} p_{i, \boldsymbol{\eta} \eta}+\frac{\partial^{2} W}{\partial\left(p_{i}\right)^{2}} p_{i, \boldsymbol{\eta}} \otimes p_{i, \boldsymbol{\eta}}+\sum_{i<j \leqslant 6} 2 \frac{\partial^{2} W}{\partial p_{i} \partial p_{j}} p_{i, \boldsymbol{\eta}} \otimes_{\text {symm }} p_{j, \boldsymbol{\eta}}\right\}
$$

Note that depending on the interatomic potential, some entries of $\partial^{2} W / \partial p_{i} \partial p_{j}$ may be zero. For instance, for the Tersoff-Brenner potential $\partial^{2} W / \partial a_{i} \partial \theta_{i} \equiv 0, i=1,3$.

A.2.3. Derivatives of $p_{i}$ with respect to the inner displacements. Let us introduce the following notation for $n=1,2$ :

$$
\begin{aligned}
& \mathscr{Q}_{n}=\mathscr{2}\left(k_{n} w^{n}\right), \quad \mathscr{Q}_{n}^{\prime}=\mathscr{Q}^{\prime}\left(k_{n} w^{n}\right), \quad \mathscr{Q}_{n}^{\prime \prime}=\mathscr{Q}^{\prime \prime}\left(k_{n} w^{n}\right) \\
& \mathscr{Q}_{n 2}=\mathscr{2}\left(k_{n} w^{n} / 2\right), \quad \mathscr{Q}_{n 2}^{\prime}=\mathscr{Q}^{\prime}\left(k_{n} w^{n} / 2\right), \quad \mathscr{Q}_{n 2}^{\prime \prime}=\mathscr{Q}^{\prime \prime}\left(k_{n} w^{n} / 2\right)
\end{aligned}
$$

The calculation of the function $2(x)$ and its derivatives is described in Appendix A.4. From Equation (20), the derivative of the deformed bond vector a expressed in the auxiliary orthonormal basis described previously can be written as

$$
\frac{\partial[\mathbf{a}]_{\tilde{\mathscr{B}}}}{\partial \boldsymbol{\eta}}=\left\{\begin{array}{c}
a_{, \boldsymbol{\eta}}^{1} \\
a_{, \boldsymbol{\eta}}^{2} \\
a_{, \boldsymbol{\eta}}^{3}
\end{array}\right\}=\left\{\begin{array}{c}
\left(\mathscr{Q}_{1}+k_{1} w^{1} \mathscr{Q}_{1}^{\prime}\right) w_{, \boldsymbol{\eta}}^{1} \\
\left(\mathscr{Q}_{2}+k_{2} w^{2} \mathscr{2}_{2}^{\prime}\right) w_{, \boldsymbol{\eta}}^{2} \\
k_{1} w^{1} \mathscr{Q}_{12}\left(\mathscr{Q}_{12}+\frac{k_{1} w^{1}}{2} \mathscr{Q}_{12}^{\prime}\right) w_{, \boldsymbol{\eta}}^{1}+\cdots \\
k_{2} w^{2} \mathscr{Q}_{22}\left(\mathscr{Q}_{22}+\frac{k_{2} w^{2}}{2} \mathscr{Q}_{22}^{\prime}\right) w_{, \boldsymbol{\eta}}^{2}
\end{array}\right\}
$$

where it can be readily seen that

$$
w_{, \boldsymbol{\eta}}^{n}=\mathbf{C V}_{n}, \quad n=1,2
$$


Note that these formulas can be applied to each undeformed lattice vector $\mathbf{A}_{i}, i=1,2,3$, and that the derivative in Equation (A15) is independent of the particular undeformed lattice vector considered, unlike $w^{n}$ (see Equation (19)).

With these expressions, the derivatives of the deformed bond lengths and angles with respect to $\boldsymbol{\eta}$ can be computed for $i=1,2,3$ as

$$
a_{i, \boldsymbol{\eta}}=\frac{1}{a_{i}}\left(a_{i}\right)^{c}\left(a_{i}\right)_{, \boldsymbol{\eta}}^{c} \text { and } \theta_{i, \boldsymbol{\eta}}=\frac{-1}{\sin \theta_{i}}\left(\cos \theta_{i}\right)_{, \boldsymbol{\eta}}
$$

where

$$
\left(\cos \theta_{i}\right)_{, \boldsymbol{\eta}}=\frac{1}{a_{j} a_{k}}\left\{\left(a_{j}\right)^{c}\left(a_{k}\right)_{, \boldsymbol{\eta}}^{c}+\left(a_{k}\right)^{c}\left(a_{j}\right)_{, \boldsymbol{\eta}}^{c}-\cos \theta_{i}\left[a_{j} a_{k, \boldsymbol{\eta}}+a_{k} a_{j, \boldsymbol{\eta}}\right]\right\}
$$

and $\{i, j, k\}$ is an even permutation of $\{1,2,3\}$. Note that $a_{i}$ denotes the length of the $i$ th bond vector, while $\left(a_{i}\right)^{c}$ denotes the $c$ th component of the $i$ th bond vector, and that according to the conventions stated in Section 2, summation on the index $c$ is implied.

A.2.4. Second derivatives of $p_{i}$ with respect to the inner displacements. Since $w_{, \eta \eta}^{n} \equiv \mathbf{0}$, the second derivative of the deformed bond vector a with respect to the inner displacements is:

$$
\frac{\partial^{2}[\mathbf{a}]_{\mathscr{B}}}{\partial \boldsymbol{\eta}^{2}}=\left\{\begin{array}{c}
k_{1}\left(2 \mathscr{Q}_{1}^{\prime}+k_{1} w^{1} \mathscr{Q}_{1}^{\prime \prime}\right) w_{, \boldsymbol{\eta}}^{1} \otimes w_{, \boldsymbol{\eta}}^{1} \\
k_{2}\left(2 \mathscr{Q}_{2}^{\prime}+k_{2} w^{2} \mathscr{Q}_{2}^{\prime \prime}\right) w_{, \boldsymbol{\eta}}^{2} \otimes w_{, \boldsymbol{\eta}}^{2} \\
k_{1}\left[\left(\mathscr{Q}_{12}+\frac{k_{1} w^{1}}{2} \mathscr{Q}_{12}^{\prime}\right)^{2}+k_{1} w^{1} \mathscr{Q}_{12}\left(\mathscr{Q}_{12}^{\prime}+\frac{k_{1} w^{1}}{4} \mathscr{Q}_{12}^{\prime \prime}\right)\right] w_{, \boldsymbol{\eta}}^{1} \otimes w_{, \boldsymbol{\eta}}^{1}+\cdots \\
k_{2}\left[\left(\mathscr{Q}_{22}+\frac{k_{2} w^{2}}{2} \mathscr{Q}_{22}^{\prime}\right)^{2}+k_{2} w^{2} \mathscr{Q}_{22}\left(\mathscr{2}_{22}^{\prime}+\frac{k_{2} w^{2}}{4} \mathscr{Q}_{22}^{\prime \prime}\right)\right] w_{, \boldsymbol{\eta}}^{2} \otimes w_{, \boldsymbol{\eta}}^{2}
\end{array}\right\}
$$

The second derivative of the bond lengths is then

$$
a_{i, \boldsymbol{\eta} \eta}=\frac{1}{a_{i}}\left[\left(a_{i}\right)_{, \boldsymbol{\eta}}^{c} \otimes\left(a_{i}\right)_{, \boldsymbol{\eta}}^{c}+\left(a_{i}\right)^{c}\left(a_{i}\right)_{, \boldsymbol{\eta} \boldsymbol{\eta}}^{c}-a_{i, \boldsymbol{\eta}} \otimes a_{i, \boldsymbol{\eta}}\right], \quad i=1,2,3
$$

For the angles, we have

$$
\theta_{i, \boldsymbol{\eta} \boldsymbol{\eta}}=\frac{-1}{\sin \theta_{i}}\left[\left(\cos \theta_{i}\right)_{, \boldsymbol{\eta} \boldsymbol{\eta}}+\left(\cos \theta_{i}\right) \theta_{i, \boldsymbol{\eta}} \otimes \theta_{i, \boldsymbol{\eta}}\right]
$$

for $i=1,2,3$. The second derivative of the cosine reads

$$
\begin{aligned}
\left(\cos \theta_{i}\right)_{, \boldsymbol{\eta}}= & \frac{1}{a_{j} a_{k}}\left[\left(\left\langle\mathbf{a}_{j} \mid \mathbf{a}_{k}\right\rangle\right)_{, \boldsymbol{\eta}}-2\left(\cos \theta_{i}\right)_{, \boldsymbol{\eta}} \otimes_{\mathrm{symm}}\left(a_{j} a_{k, \boldsymbol{\eta}}+a_{k} a_{j, \boldsymbol{\eta}}\right)\right. \\
& \left.-\cos \theta_{i}\left(a_{j} a_{k, \boldsymbol{\eta} \eta}+a_{k} a_{j, \boldsymbol{\eta} \eta}+2 a_{j, \boldsymbol{\eta}} \otimes_{\mathrm{symm}} a_{k, \boldsymbol{\eta}}\right)\right]
\end{aligned}
$$

where

$$
\left(\left\langle\mathbf{a}_{j} \mid \mathbf{a}_{k}\right\rangle\right)_{, \boldsymbol{\eta} \eta}=\left(a_{j}\right)^{c}\left(a_{k}\right)_{, \boldsymbol{\eta} \boldsymbol{\eta}}^{c}+\left(a_{k}\right)^{c}\left(a_{j}\right)_{, \boldsymbol{\eta} \boldsymbol{\eta}}^{c}+2\left(a_{j}\right)_{, \boldsymbol{\eta}}^{c} \otimes_{\mathrm{symm}}\left(a_{k}\right)_{, \boldsymbol{\eta}}^{c}
$$

and $\{i, j, k\}$ is an even permutation of $\{1,2,3\}$. 


\section{A.3. Derivatives of $a_{i}$ and $\theta_{i}$ with respect to the strain measures}

The present appendix describes the calculation of the derivatives of the derived strain measures $a_{i}$ and $\theta_{i}$ with respect to the strain measures $\mathbf{C}$ and $\mathscr{K}$. Recall that in Appendix A.1 the derivatives of the principal curvatures and directions with respect to the strain measures were presented. These results are required in the formulas below.

It can be shown that

$$
\frac{\partial w^{n}}{\partial \mathbf{C}}=C_{A B} A^{A} \frac{\partial\left(V_{n}\right)^{B}}{\partial \mathbf{C}}+\mathbf{A} \otimes_{\text {symm }} \mathbf{V}_{n} \quad \text { and } \quad \frac{\partial w^{n}}{\partial \mathscr{K}}=C_{A B} A^{A} \frac{\partial\left(V_{n}\right)^{B}}{\partial \mathscr{K}}
$$

for $n=1,2$. Then, for the deformed bond vector we have

$$
\frac{\partial[\mathbf{a}]_{\tilde{\mathscr{B}}}}{\partial \bullet}=\left\{\begin{array}{c}
\mathscr{Q}_{1} \frac{\partial w^{1}}{\partial \bullet}+w^{1} \mathscr{Q}_{1}^{\prime}\left(w^{1} \frac{\partial k_{1}}{\partial \bullet}+k_{1} \frac{\partial w^{1}}{\partial \bullet}\right) \\
\mathscr{Q}_{2} \frac{\partial w^{2}}{\partial \bullet}+w^{2} \mathscr{Q}_{2}^{\prime}\left(w^{2} \frac{\partial k_{2}}{\partial \bullet}+k_{2} \frac{\partial w^{2}}{\partial \bullet}\right) \\
\frac{1}{2}\left[w^{1} \mathscr{Q}_{12}^{2}\left(w^{1} \frac{\partial k_{1}}{\partial \bullet}+2 k_{1} \frac{\partial w^{1}}{\partial \bullet}\right)+k_{1}\left(w^{1}\right)^{2} \mathscr{Q}_{12} \mathscr{Q}_{12}^{\prime}\left(w^{1} \frac{\partial k_{1}}{\partial \bullet}+k_{1} \frac{\partial w^{1}}{\partial \bullet}\right)+\cdots\right. \\
\left.w^{2} \mathscr{Q}_{22}^{2}\left(w^{2} \frac{\partial k_{2}}{\partial \bullet}+2 k_{2} \frac{\partial w^{2}}{\partial \bullet}\right)+k_{2}\left(w^{2}\right)^{2} \mathscr{Q}_{22} \mathscr{Q}_{22}^{\prime}\left(w^{2} \frac{\partial k_{2}}{\partial \bullet}+k_{2} \frac{\partial w^{2}}{\partial \bullet}\right)\right]
\end{array}\right\}
$$

where $\bullet$ denotes either $\mathbf{C}$ or $\mathscr{K}$. Finally, similarly to the case of the derivatives with respect to the inner displacements, we have for each bond vector and angle $i=1,2,3$

$$
\frac{\partial a_{i}}{\partial \bullet}=\frac{1}{a_{i}}\left(a_{i}\right)^{c} \frac{\partial\left(a_{i}\right)^{c}}{\partial \bullet}
$$

and

$$
\frac{\partial \theta_{i}}{\partial \bullet}=\frac{-1}{\sin \theta_{i} a_{j} a_{k}}\left\{\left(a_{j}\right)^{c} \frac{\partial\left(a_{k}\right)^{c}}{\partial \bullet}+\left(a_{k}\right)^{c} \frac{\partial\left(a_{j}\right)^{c}}{\partial \bullet}-\cos \theta_{i}\left[a_{j} \frac{\partial a_{k}}{\partial \bullet}+a_{k} \frac{\partial a_{j}}{\partial \bullet}\right]\right\}
$$

where $\{i, j, k\}$ is an even permutation of $\{1,2,3\}$.

\section{A.4. Calculation of $2(x), \mathscr{Q}^{\prime}(x)$ and $\mathscr{2}^{\prime \prime}(x)$}

The calculation of the functions

$$
\mathscr{2}(x)=\frac{\sin x}{x}, \quad \mathscr{Q}^{\prime}(x)=\frac{\cos x-\mathscr{2}(x)}{x} \quad \text { and } \quad \mathscr{Q}^{\prime \prime}(x)=-\frac{\sin x+2 \mathscr{Q}^{\prime}(x)}{x}
$$

must be performed with care when $x \ll 1$ to prevent numerical roundoff errors. Note that the limits of the above functions when $x \rightarrow 0$ exist, and therefore they can be extended by continuity at $x=0\left(\mathscr{Q}(0)=1, \mathscr{Q}^{\prime}(0)=0\right.$ and $\left.\mathscr{Q}^{\prime \prime}(0)=-1 / 3\right)$. This situation occurs when one of the principal curvatures is zero, for instance when the deformed body is a cylinder. In the computer implementation, these functions are evaluated by a tenth order Taylor series expansion, which is very accurate for the range of finite values of $x$ encountered in the calculations, and faster to evaluate than the trigonometric functions. 


\section{APPENDIX B: VARIATIONS OF THE STRAIN MEASURES}

\section{B.1. Continuum problem}

The variations of the strain measures $\delta \mathbf{C}$ and $\delta \mathscr{K}$ corresponding to $\delta \Phi$ are describes next. Since the undeformed configuration is fixed, we have $\delta \Phi=\delta \varphi \circ \varphi_{0}$. For this reason, we will express $\delta \mathbf{C}$ and $\delta \mathscr{K}$ in terms of the variations of the deformed configuration $\varphi$.

Recalling Equations (2) and (7), we have

$$
\delta g_{\alpha \beta}=\left(g_{\alpha}\right)^{a} \frac{\partial(\delta \varphi)^{a}}{\partial \xi^{\beta}}+\frac{\partial(\delta \varphi)^{b}}{\partial \xi^{\alpha}}\left(g_{\beta}\right)^{b}
$$

and from Equation (9), the variation of the Green deformation tensor can be written as

$$
\delta C_{A B}=\left(T \varphi_{0}^{-1}\right)^{\alpha}{ }_{A}\left(T \varphi_{0}^{-1}\right)^{\beta}{ }_{A} \delta g_{\alpha \beta}
$$

For the curvature, it follows from Equation (12) that

$$
\delta k_{\alpha \beta}=(\delta n)^{a}\left(g_{\alpha, \beta}\right)^{a}+n^{b} \frac{\partial^{2}(\delta \varphi)^{b}}{\partial \xi^{\alpha} \partial \xi^{\beta}}
$$

where

$$
\delta \mathbf{n}=\frac{1}{\left\|\mathbf{g}_{1} \times \mathbf{g}_{2}\right\|}\left[\frac{\partial(\delta \boldsymbol{\varphi})}{\partial \xi^{1}} \times \mathbf{g}_{2}+\mathbf{g}_{1} \times \frac{\partial(\delta \boldsymbol{\varphi})}{\partial \xi^{2}}-\delta\left\|\mathbf{g}_{1} \times \mathbf{g}_{2}\right\| \mathbf{n}\right]
$$

and noting that $\left\|\mathbf{g}_{1} \times \mathbf{g}_{2}\right\|^{2}=\operatorname{det}\left(g_{\alpha \beta}\right)=g_{11} g_{22}-g_{12}^{2}$, it follows:

$$
\delta\left\|\mathbf{g}_{1} \times \mathbf{g}_{2}\right\|=\frac{1}{2\left\|\mathbf{g}_{1} \times \mathbf{g}_{2}\right\|}\left(\delta g_{11} g_{22}+g_{11} \delta g_{22}-2 g_{12} \delta g_{12}\right)
$$

Finally, the variation of the pull-back of the curvature tensor is

$$
\delta \mathscr{K}_{A B}=\left(T \varphi_{0}^{-1}\right)^{\alpha}{ }_{A}\left(T \varphi_{0}^{-1}\right)^{\beta}{ }_{B} \delta k_{\alpha \beta}
$$

\section{B.2. Discrete problem}

The analogues of the variations of the strain measures in the discrete finite element problem are the derivatives of the strain measures with respect to the degrees of freedom. These are needed in the calculation of the internal forces, see Section 4.2. It follows from Equations (7) and (2) that

$$
\frac{\partial g_{\alpha \beta}}{\partial \boldsymbol{\varphi}_{I}^{e}}=N_{I, \alpha} \mathbf{g}_{\beta}+N_{I, \beta} \mathbf{g}_{\alpha}
$$

Then, from Equations (9) and (48), the derivatives of the Green deformation tensor with respect to the nodal degrees of freedom can be written as

$$
\frac{\partial C_{A B}}{\partial \boldsymbol{\varphi}_{I}^{e}}=\left[\left(T \varphi_{0}^{e}\right)^{-1}\right]^{\alpha}{ }_{A}\left[\left(T \varphi_{0}^{e}\right)^{-1}\right]_{B}^{\beta} \frac{\partial g_{\alpha \beta}}{\partial \varphi_{I}^{e}}
$$


For the covariant components of the curvature tensor defined in Equation (12), and recalling Equation (47), we have

$$
\frac{\partial k_{\alpha \beta}}{\partial \boldsymbol{\varphi}_{I}^{e}}=\left(g_{\alpha, \beta}\right)^{a} \frac{\partial n^{a}}{\partial \boldsymbol{\varphi}_{I}^{e}}+N_{I, \alpha \beta} \mathbf{n}
$$

where it can be readily seen from Equations (11) and (2) that

$$
\frac{\partial n^{a}}{\partial \boldsymbol{\varphi}_{I}^{e}}=\frac{1}{\left\|\mathbf{g}_{1} \times \mathbf{g}_{2}\right\|}\left[\mathbf{i}_{a} \times\left(N_{I, 2} \mathbf{g}_{1}-N_{I, 1} \mathbf{g}_{2}\right)-n^{a} \frac{\partial\left\|\mathbf{g}_{1} \times \mathbf{g}_{2}\right\|}{\partial \boldsymbol{\varphi}_{I}^{e}}\right]
$$

and, as for Equation (B5), we have

$$
\frac{\partial\left\|\mathbf{g}_{1} \times \mathbf{g}_{2}\right\|}{\partial \boldsymbol{\varphi}_{I}^{e}}=\frac{1}{\left\|\mathbf{g}_{1} \times \mathbf{g}_{2}\right\|}\left[\left(N_{I, 1} g_{22}-N_{I, 2} g_{12}\right) \mathbf{g}_{1}-\left(N_{I, 1} g_{12}-N_{I, 2} g_{11}\right) \mathbf{g}_{2}\right]
$$

The final result for the components of the pull-back of the curvature tensor is

$$
\frac{\partial \mathscr{K}_{A B}}{\partial \boldsymbol{\varphi}_{I}^{e}}=\left[\left(T \varphi_{0}^{e}\right)^{-1}\right]_{A}^{\alpha}\left[\left(T \varphi_{0}^{e}\right)^{-1}\right]_{B}^{\beta} \frac{\partial k_{\alpha \beta}}{\partial \varphi_{I}^{e}}
$$

\section{REFERENCES}

1. Yakobson BI, Brabec CJ, Bernholc J. Nanomechanics of carbon tubes: Instabilities beyond the linear response. Physical Review Letters 1996; 76(14):2511-2514.

2. Belytschko T, Xiao SP, Schatz GC, Ruoff RS. Atomistic simulation of nanotube fracture. Physical Review B 2002; 65(235430).

3. Hertel T, Walkup RE, Avouris P. Deformation of carbon nanotubes by surface van der Waals forces. Physical Review B 1998; 58(20):13870-13873.

4. Rochefort A, Avouris P, Lesage F, Salahub DR. Electrical and mechanical properties of distorted carbon nanotubes. Physical Review B 1999; 60(19):13824-13830.

5. Zhou G, Duan W, Gu B. First-principles study on morphology and mechanical properties of single-walled carbon nanotube. Chemical Physics Letters 2001; 333:344-349.

6. Sánchez-Portal D, Artacho E, Soler J, Rubio A, Ordejón P. Ab initio structural, elastic, and vibrational properties of carbon nanotubes. Physical Review B 1999; 59(19):12678-12688.

7. Arroyo M, Belytschko T. Membranes and rods from lattice films and chains: modelling and computations. Proceedings of the Second MIT Conference of Computational Fluid and Solid Mechanics, 2003.

8. Iijima S, Brabec C, Maiti A, Bernholc J. Structural flexibility of carbon nanotubes. Journal of Chemical Physics 1996; 104(5):2089-2092.

9. Falvo MR, Clary GJ, Taylor RM, Chi V, Brooks FP, Washburn S, Superfine R. Bending and buckling of carbon nanotubes under large strain. Nature 1997; 389:582-584.

10. Walters DA, Ericson LM, Casavant MJ, Liu J, Colbert DT, Smith KA, Smalley RE. Elastic strain of freely suspended single-wall carbon nanotube ropes. Applied Physics Letters 1999; 74(25):3803-3805.

11. Tombler TW, Zhou C, Alexseyev L, Kong J, Dai H, Liu L, Jayanthi CS, Tang M, Wu SY. Reversible electromechanical characteristics of carbon nanotubes under local-probe manipulation. Nature 2000; 405: 769-772.

12. Rueckes T, Kim K, Joselevich E, Tseng GY, Cheung CL, Lieber CM. Carbon nanotube-based nonvolatile random access memory for molecular computing. Science 2000; 289:94-97.

13. Chopra NG, Benedict LX, Crespi VH, Cohen ML, Louie SG, Zettl A. Fully collapsed carbon nanotubes. Nature 1995; 377:135-138.

14. Yu M, Kowalewski T, Ruoff RS. Structural analysis of collapsed, and twisted and collapsed, multiwalled carbon nanotubes by atomic force microscopy. Physical Review Letters 2001; 86(1):87-90.

15. Sohlberg K, Sumpter BG, Tuzun RE, Noid DW. Continuum methods of mechanics as a simplified approach to structural engineering of nanostructures. Nanotechnology 1998; 9:30-36.

16. Ou-Yang Zhong-can, Zhao-Bin Su, and Chui-Lin Wang. Coil formation in multishell carbon nanotubes: competition between curvature elasticity and interlayer adhesion. Physical Review Letters 1997; 78(21): 4055-4058. 
17. Govindjee S, Sackman JL. On the use of continuum mechanics to estimate the properties of carbon nanotubes. Solid State Communication 1999; 110:227-230.

18. Ru CQ. Effective bending stiffness of carbon nanotubes. Physical Review B 2000; 62(15):9973-9976.

19. Ru CQ. Axially compressed buckling of a doublewalled carbon nanotube embedded in an elastic medium. Journal of the Mechanics and Physics of Solids 2001; 49:1265-1279.

20. Popov VN, Van Doren VE, Balkanski M. Elastic properties of single-walled carbon nanotubes. Physical Review B 2000; 61(4):3078-3084.

21. Lourie O, Cox DM, Wagner HD. Buckling and collapse of embedded carbon nanotubes. Physical Review Letters 1998; 81(8):1638-1641.

22. Yu M, Lourie O, Dyer MJ, Moloni K, Kelly TF, Ruoff RS. Strength and breaking mechanism of multiwalled carbon nanotubes under tensile load. Science 2000; 287:637-640.

23. Arroyo M, Belytschko T. Finite crystal elasticity of carbon nanotubes based on the exponential Cauchy-Born rule. 2003, submitted.

24. Arroyo M, Belytschko T. Nonlinear mechanical response and rippling of thick multi-walled carbon nanotubes. accepted for publication in Physical Review Letters.

25. Gao XL, Li K, Roy AK. Finite deformation continuum model for energetics of carbon nanotubes. Proceedings of the 44th AIAA/ASME/ASCE/AHS/ASC SDM Conference 2003.

26. Pantano A, Parks DM, Boyce MC. Mechanics of deformation of single and multi-wall carbon nanotubes. 2003, submitted.

27. Friesecke G, James RD. A scheme for the passage from atomic to continuum theory for thin films, nanotubes and nanorods. Journal of the Mechanics and Physics of Solids 2000; 48:1519-1540.

28. Qian D, Wagner GJ, Liu WK, Yu MF, Ruoff RS. Mechanics of carbon nanotubes. Applied Mechanics Reviews 2002; 55(6):495-553.

29. Stakgold I. The Cauchy relations in a molecular theory of elasticity. Quarterly Journal of Applied Mathematics $1950 ; 8(2): 169-186$.

30. Ericksen JL. Nonlinear elasticity of diatomic crystals. International Journal of Solids and Structures 1970; 6:951-957.

31. Martin JW. Many-body forces in metals and the Brugger elastic constants. Journal of Physics C 1975; 8: $2837-2857$.

32. Cousins CSG. Inner elasticity. Journal of Physics C 1978; 11:4867-4879.

33. Milstein F. In Mechanics of Solids. Hopkins HG, Sewell MJ (eds). Pergamon: Oxford, 1982.

34. Weiner JH. Statistical Mechanics of Elasticity. Wiley: New York, 1983.

35. Ericksen JL. Phase Transformations and Material Instabilities in Solids, Gurtin ME (ed.), Chapter The Cauchy and Born hypotheses for crystals, Academic Press, January 1984, pp. 61-77.

36. Friesecke G, Theil F. Validity and failure of the Cauchy-Born hypothesis in a two-dimensional mass-spring lattice. Journal of Nonlinear Science 2002; 12(5):445-478.

37. Hill R. On the elasticity and stability of perfect crystals at finite strains. Mathematical Proceedings of the Cambridge Philosophical Society 1975; 77:225-240.

38. Cousins CSG. Inner elasticity and the higher-order elasticity of some diamond and graphite allotropes. Ph.D. Thesis, University of Exeter, 2001.

39. Zanzotto G. The Cauchy-Born hypothesis, non-linear elasticity and mechanical twinning in crystals. Acta Crystallographica A 1996; 52:839-849.

40. Tadmor EB, Ortiz M, Phillips R. Quasicontinuum analysis of defects in solids. Philosophical Magazine A 1996; 73(6):1529-1563

41. Shenoy VB, Miller R, Tadmor EB, Rodney D, Phillips R, Ortiz M. An adaptive finite element approach to atomic-scale mechanics - the quasicontinuum method. Journal of the Mechanics and Physics of Solids 1999; 47:611-642.

42. Tadmor WB, Smith GS, Bernstein N, Kaxiras E. Mixed finite element and atomistic formulation for complex crystals. Physical Review B 1999; 59(1):235-245.

43. Smith GS, Tadmor EB, Kaxiras E. Multiscale simulation of loading and electrical resistance in silicon nanoindentation. Physical Review Letters 2000; 84(6):1260-1263.

44. Arroyo M, Belytschko T. An atomistic-based finite deformation membrane for single layer crystalline films. Journal of the Mechanics and Physics of Solids 2002; 50(9):1941-1977.

45. Qian D, Liu WK, Ruoff RS. Mechanics of $\mathrm{C}_{60}$ in nanotubes. Journal of Physical Chemistry B 2001; 105: 10753-10758.

46. Zhang P, Huang Y, Gao H, Hwang KC. Fracture nucleation in single-wall carbon nanotubes under tension: a continuum analysis incorporating interatomic potentials. Journal of Applied Mechanics 2002; 69:454-458.

47. Marsden JE, Hughes TJR. Mathematical Foundations of Elasticity. Prentice-Hall: Englewood Cliffs, NJ, 1983.

48. Manfredo P. do Carmo. Differential Geometry of Curves and Surfaces. Prentice-Hall: Englewood Cliffs, NJ, 1976.

49. Dierkes U, Hildebrandt S, Küster A, Wohlrab O. Minimal surfaces I, Comprehensive Studies in Mathematics, vol. 295. Springer-Verlag: Berlin, 1992. 
50. Arroyo M, Belytschko T. A finite deformation membrane based on inter-atomic potentials for the transverse mechanics of nanotubes. Mechanics of Materials 2003; 35(3-6):193-215.

51. Saito R, Fujita M, Dresselhaus G, Dresselhaus MS. Electronic structure of chiral graphene tubules. Applied Physics Letters 1992; 60(18):2204-2206.

52. Tadmor EB, Waghmare UV, Smith GS, Kaxiras E. Polarization switching in $\mathrm{PbTiO}_{3}$ : an ab initio finite element simulation. Acta Materialia 2002; 50(11):2989-3002.

53. Brenner DW. Empirical potential for hydrocarbons for use in simulating chemical vapor deposition of diamond films. Physical Review B 1990; 42(15):9458-9471.

54. Tersoff J. New empirical approach for the structure and energy of covalent systems. Physical Review B 1988; 37(12):6991-7000.

55. Cornwell CF, Willie LT. Elastic properties of single-walled carbon nanotubes in compression. Solid State Communications 1997; 101(8):555-558.

56. Girifalco LA, Hodak M, Lee RS. Carbon nanotubes, buckyballs, ropes, and a universal graphitic potential. Physical Review B 2000; 62(19):13104-13110.

57. Belytschko T, Liu WK, Moran B. Nonlinear Finite Elements for Continua and Structures. Wiley, 2000.

58. Cirak F, Ortiz M, Schröder P. Subdivision surfaces: a new paradigm for thin-shell finite-element analysis. International Journal for Numerical Methods in Engineering 2000; 47:2039-2072.

59. Krysl P, Belytschko T. Analysis of thin shells by the element-free Galerkin method. International Journal of Solids and Structures 1996; 33(20-22):3057-3078.

60. Gilbert JC, Nocedal J. Global convergence properties of conjugate gradient methods for optimization. SIAM Journal on Optimization 1992; 2(1).

61. Liu DC, Nocedal J. On the limited memory method for large scale optimization. Mathematical Programming B 1989; 45(3):503-528.

62. Dacorogna B. Direct Methods in the Calculus of Variations, Applied Mathematical Sciences, vol. 78. Berlin: Springer-Verlag, 1989.

63. Conti S, DeSimone A, Dolzmann G. Soft elastic response of stretched sheets of nematic elastomers: a numerical study. Journal of the Mechanics and Physics of Solids 2002; 50(7):1431-1451.

64. Ruoff RS, Lorents DC. Mechanical and thermal properties of carbon nanotubes. Carbon 1995; 33(7):925-930.

65. Kuzumaki T, Hayashi T, Ichinose H, Miyazawa K, Ito K, Ishida Y. In-situ observed deformation of carbon nanotubes. Philosophical Magazine A 1998; 77(6):1461-1469.

66. Poncharal P, Wang ZL, Ugarte D, de Heer WA. Electrostatic deflections and electromechanical resonances of carbon nanotubes. Science 1999; 283:1513-1516.

67. Bažant ZP, Cedolin L. Stability of Structures. Oxford University Press: Oxford, 1991.

68. Fox RL, Kapoor MP. Rates of change of eigenvalues and eigenvectors. AIAA Journal 1968; 6(12):2426-2429.

69. Friswell MI. The derivatives of repeated eigenvalues and their associated eigenvectors. Journal of Vibration and Acoustics (ASME) 1996; 118:390-397. 